Pacific

Journal of

Mathematics

\title{
HERMITIAN CATEGORIES, EXTENSION OF SCALARS AND SYSTEMS OF SESQUILINEAR FORMS
}

Eva Bayer-Fluckiger, Uriya A. First and Daniel A. Moldovan 


\title{
HERMITIAN CATEGORIES, EXTENSION OF SCALARS AND SYSTEMS OF SESQUILINEAR FORMS
}

\author{
Eva Bayer-Fluckiger, Uriya A. First and Daniel A. Moldovan
}

We prove that the category of systems of sesquilinear forms over a given hermitian category is equivalent to the category of unimodular 1-hermitian forms over another hermitian category. The sesquilinear forms are not required to be unimodular or defined on a reflexive object (i.e., the standard map from the object to its double dual is not assumed to be bijective), and the forms in the system can be defined with respect to different hermitian structures on the given category. This extends an earlier result of the first and third authors.

We use the equivalence to define a Witt group of sesquilinear forms over a hermitian category and to generalize results such as Witt's cancellation theorem, Springer's theorem, the weak Hasse principle, and finiteness of genus to systems of sesquilinear forms over hermitian categories.

\section{Introduction}

Quadratic and hermitian forms were studied extensively by various authors, who have developed a rich array of tools to study them. It is well known that in many cases (e.g., over fields), the theory of sesquilinear forms can be reduced to the theory of hermitian forms (e.g., see [Riehm 1974; Riehm and Shrader-Frechette 1976] and works based on them). In [Bayer-Fluckiger and Moldovan 2014], an explanation of this reduction was provided in the form of an equivalence between the category of sesquilinear forms over a ring and the category of unimodular 1-hermitian forms over a special hermitian category.

In this paper, we extend the equivalence of [Bayer-Fluckiger and Moldovan 2014] to hermitian categories, and, moreover, improve it in such a way that it applies to systems of sesquilinear forms in hermitian categories that admit nonreflexive objects (see Section 2). That is, we prove that the category of systems of sesquilinear forms over a hermitian category $\mathscr{b}$ is equivalent to the category of unimodular 1-hermitian

First is partially supported by an Israel-US BSF grant \#2010/149 and an ERC grant \#226135. Moldovan was partially supported by the Swiss National Science Foundation, grant 200020-109174/1. MSC2010: 11E39, 11E81.

Keywords: sesquilinear forms, hermitian forms, systems of sesquilinear forms, hermitian categories, additive categories, $K$-linear categories, scalar extension, Witt group. 
forms over anther hermitian category $\mathfrak{C}^{\prime}$. The sesquilinear forms are not required to be unimodular or defined on a reflexive object, and the forms in the system can be defined with respect to different hermitian structures on the category $\mathscr{b}$.

Using the equivalence, we present a notion of a Witt group of sesquilinear forms, which is analogous to the standard Witt group of hermitian forms over rings with involution (e.g., see [Knus 1991; Scharlau 1985]). We also extend various results (Witt's cancellation theorem, Springer's theorem, finiteness of genus, the Hasse principle, etc.) to systems of sesquilinear forms over hermitian categories (and in particular to systems of sesquilinear forms over rings with a family of involutions).

Sections 1 and 2 recall the basics of sesquilinear forms over rings and hermitian categories, respectively. In Section 3, we prove the equivalence of the category of sesquilinear forms over a given hermitian category to a category of unimodular 1-hermitian forms over another hermitian category, and in Section 4 we extend this result to systems of sesquilinear forms. Section 5 presents applications of the equivalence.

\section{Sesquilinear and hermitian forms}

Let $A$ be a ring. An involution on $A$ is an additive map $\sigma: A \rightarrow A$ such that $\sigma(a b)=\sigma(b) \sigma(a)$ for all $a, b \in A$ and $\sigma^{2}=\operatorname{id}_{A}$. Let $V$ be a right $A$-module. A sesquilinear form over $(A, \sigma)$ is a biadditive map $s: V \times V \rightarrow A$ satisfying $s(x a, y b)=\sigma(a) s(x, y) b$ for all $x, y \in V$ and $a, b \in A$. The pair $(V, s)$ is also called a sesquilinear form in this case. ${ }^{1}$ The orthogonal sum of two sesquilinear forms $(V, s)$ and $\left(V^{\prime}, s^{\prime}\right)$ is defined to be $\left(V \oplus V^{\prime}, s \oplus s^{\prime}\right)$ where $s \oplus s^{\prime}$ is given by

$$
\left(s \oplus s^{\prime}\right)\left(x \oplus x^{\prime}, y \oplus y^{\prime}\right)=s(x, y)+s^{\prime}\left(x^{\prime}, y^{\prime}\right)
$$

for all $x, y \in V$ and $x^{\prime}, y^{\prime} \in V^{\prime}$. Two sesquilinear forms $(V, s)$ and $\left(V^{\prime}, s^{\prime}\right)$ are called isometric if there exists an isomorphism of $A$-modules $f: V \stackrel{\sim}{\longrightarrow} V^{\prime}$ such that $s^{\prime}(f(x), f(y))=s(x, y)$ for all $x, y \in V$.

Let $V^{*}=\operatorname{Hom}_{A}(V, A)$. Then $V^{*}$ has a right $A$-module structure given by $(f \cdot a)(x)=\sigma(a) f(x)$ for all $f \in V^{*}, a \in A$. We say that $V$ is reflexive if the homomorphism of right $A$-modules $\omega_{V}: V \rightarrow V^{* *}$ defined by $\omega_{V}(x)(f)=\sigma(f(x))$ for all $x \in V, f \in V^{*}$ is bijective.

A sesquilinear space $(V, s)$ over $(A, \sigma)$ induces two homomorphisms of right $A$-modules $s_{\ell}, s_{r}: V \rightarrow V^{*}$, called the left and right adjoint of $s$, respectively. They are given by $s_{\ell}(x)(y)=s(x, y)$ and $s_{r}(x)(y)=\sigma(s(y, x))$ for all $x, y \in V$. Observe that $s_{r}=s_{\ell}^{*} \omega_{V}$ and $s_{\ell}=s_{r}^{*} \omega_{V}$. The form $s$ is called unimodular if $s_{r}$ and $s_{\ell}$ are isomorphisms. In this case, $V$ must be reflexive.

\footnotetext{
${ }^{1}$ Some texts use the term sesquilinear space.
} 
Let $\epsilon= \pm 1$. A sesquilinear form $(V, s)$ over $(A, \sigma)$ is called $\epsilon$-hermitian if $\sigma(s(x, y))=\epsilon s(y, x)$ for all $x, y \in V$, that is, if $s_{r}=\epsilon s_{\ell}$. A 1-hermitian form is also called a hermitian form.

There exists a classical notion of Witt group for unimodular $\epsilon$-hermitian forms over $(A, \sigma)$ (e.g., see [Knus 1991]): denote by $\mathrm{WG}^{\epsilon}(A, \sigma)$ the Grothendieck group of isometry classes of unimodular $\epsilon$-hermitian forms $(V, s)$ over $(A, \sigma)$, with $V$ finitely generated projective, addition being the orthogonal sum. A unimodular $\epsilon$-hermitian form over $(A, \sigma)$ is called hyperbolic if it is isometric to $\left(V \oplus V^{*}, \mathbb{U}_{V}^{\epsilon}\right)$ for some finitely generated projective right $A$-module $V$, where $\mathbb{U}_{V}^{\epsilon}$ is defined by

$$
\mathbb{H}_{V}^{\epsilon}(x \oplus f, y \oplus g)=f(y)+\epsilon \sigma(g(x)) \text { for all } x, y \in V, f, g \in V^{*} .
$$

We let $\mathbb{W}_{V}=\mathbb{W}_{V}^{1}$. The quotient of $\mathrm{WG}^{\epsilon}(A, \sigma)$ by the subgroup generated by the unimodular $\epsilon$-hermitian hyperbolic forms is called the Witt group of unimodular $\epsilon$-hermitian forms over $(A, \sigma)$ and is denoted by $\mathrm{W}^{\epsilon}(A, \sigma)$.

We denote by $\operatorname{Sesq}(A, \sigma)$ and $\operatorname{UH}^{\epsilon}(A, \sigma)$ the categories of sesquilinear and unimodular $\epsilon$-hermitian forms over $(A, \sigma)$, respectively. The morphisms of these categories are (bijective) isometries. For simplicity, we let $\operatorname{UH}(A, \sigma):=\operatorname{UH}^{1}(A, \sigma)$.

\section{Hermitian categories}

This section recalls some basic notions about hermitian categories, as presented in [Scharlau 1985] (see also [Knus 1991; Quebbemann et al. 1979]).

2A. Preliminaries. Recall that a hermitian category consists of a triple $(\mathscr{b}, *, \omega)$, where $\mathscr{C}$ is an additive category, $*: \mathscr{C} \rightarrow \mathscr{C}$ is a contravariant functor and $\omega=$ $\left(\omega_{C}\right)_{C \in \mathscr{C}}: \mathrm{id} \rightarrow * *$ is a natural transformation satisfying $\omega_{C}^{*} \omega_{C^{*}}=\operatorname{id}_{C^{*}}$ for all $C \in \mathscr{C}$. In this case, the pair $(*, \omega)$ is called a hermitian structure on $\mathscr{C}$. It is customary to assume that $\omega$ is a natural isomorphism rather than a natural transformation. Such hermitian categories will be called reflexive. In general, an object $C \in \mathscr{C}$ for which $\omega_{C}$ is an isomorphism is called reflexive, so the category $\mathscr{b}$ is reflexive precisely when all its objects are reflexive. We will often drop $*$ and $\omega$ from the notation and use these symbols to denote the functor and natural transformation associated with any hermitian category under discussion.

A sesquilinear form over the category $\mathscr{C}$ is a pair $(C, s)$ with $C \in \mathscr{C}$ and $s: C \rightarrow C^{*}$. A sesquilinear form $(C, s)$ is called unimodular if $s$ and $s^{*} \omega_{C}$ are isomorphisms. (If $C$ is reflexive, then $s$ is bijective if and only if $s^{*} \omega_{C}$ is bijective.) Let $\epsilon= \pm 1$. A sesquilinear form $(C, s)$ is called $\epsilon$-hermitian if $s=\epsilon s^{*} \omega_{C}$. For brevity, 1-hermitian forms are often called hermitian forms. Orthogonal sums of forms are defined in the obvious way. Let $(C, s)$ and $\left(C^{\prime}, s^{\prime}\right)$ be two sesquilinear forms over $\mathscr{C}$. An isometry from $(C, s)$ to $\left(C^{\prime}, s^{\prime}\right)$ is an isomorphism $f: C \stackrel{\sim}{\longrightarrow} C^{\prime}$ satisfying $s=f^{*} s^{\prime} f$. In 
this case, $(C, s)$ and $\left(C^{\prime}, s^{\prime}\right)$ are said to be isometric. We let Sesq( $(\mathscr{C})$ stand for the category of sesquilinear forms over $\mathscr{C}$ with isometries as morphisms.

Denote by $\mathrm{UH}^{\epsilon}(\mathscr{C})$ the category of unimodular $\epsilon$-hermitian forms over $\mathscr{C}$. The morphisms are isometries. For brevity, let $\mathrm{UH}(\mathscr{C}):=\mathrm{UH}^{1}(\mathscr{C})$. The hyperbolic unimodular $\epsilon$-hermitian forms over $\mathscr{C}$ are the forms isometric to $\left(Q \oplus Q^{*}, \mathbb{T}_{Q}^{\epsilon}\right)$, where $Q$ is any reflexive object in $\mathscr{C}$ and $\mathbb{U}_{Q}^{\epsilon}$ is given by

$$
\mathbb{H}_{Q}^{\epsilon}=\left[\begin{array}{cc}
0 & \operatorname{id}_{Q^{*}} \\
\epsilon \omega_{Q} & 0
\end{array}\right]: Q \oplus Q^{*} \rightarrow\left(Q \oplus Q^{*}\right)^{*}=Q^{*} \oplus Q^{* *}
$$

Again, let $\mathbb{M}_{Q}=\mathbb{\boxplus}_{Q}^{1}$. The quotient of $\mathrm{WG}^{\epsilon}(\mathscr{C})$, the Grothendieck group of isometry classes of unimodular $\epsilon$-hermitian forms over $\mathscr{C}$ (with respect to the orthogonal sum), by the subgroup generated by the hyperbolic forms is called the Witt group of unimodular $\epsilon$-hermitian forms over $\mathscr{C}$ and is denoted by $\mathrm{W}^{\epsilon}(\mathscr{C})$. For brevity, set $\mathrm{W}(\mathscr{C})=\mathrm{W}^{1}(\mathfrak{C})$.

Example 2.1. Let $(A, \sigma)$ be a ring with involution. If we take $\mathscr{b}$ to be Mod- $A$, the category of right $A$-modules, and define $*$ and $\omega$ as in Section 1 , then $\mathscr{b}$ becomes a hermitian category. Furthermore, the sesquilinear forms $(M, s)$ over $(A, \sigma)$ correspond to the sesquilinear forms over $\mathscr{C}$ via $(M, s) \mapsto\left(M, s_{r}\right)$. This correspondence gives rise to isomorphisms of categories $\operatorname{Sesq}(A, \sigma) \cong \operatorname{Sesq}(\mathscr{C})$ and $\mathrm{UH}^{\epsilon}(A, \sigma) \cong \mathrm{UH}^{\epsilon}(\mathscr{C})$. Now let $\mathscr{C}$ be a subcategory of Mod- $A$ such that $M \in \mathscr{C}$ implies $M^{*} \in \mathscr{C}$. Then $\mathscr{C}$ is still a hermitian category, and is reflexive if and only if $\mathscr{C}$ consists of reflexive $A$-modules (as defined in Section 1). For example, this happens if $\mathscr{C}=\mathscr{P}(A)$, the category of projective $A$-modules of finite type. In this case, the Witt group $\mathrm{W}^{\epsilon}(\mathscr{C})=\mathrm{W}^{\epsilon}(\mathscr{P}(A))$ is isomorphic to $\mathrm{W}^{\epsilon}(A, \sigma)$.

2B. Duality-preserving functors. Let $\mathscr{C}$ and $\mathscr{C}^{\prime}$ be two hermitian categories. A duality-preserving functor from $\mathscr{C}$ to $\mathscr{C}^{\prime}$ is an additive functor $F: \mathscr{C} \rightarrow \mathscr{C}^{\prime}$ together with a natural isomorphism $i=\left(i_{M}\right)_{M \in \mathscr{C}}: F * \rightarrow * F$. This means that for any $M \in \mathscr{C}$, there exists an isomorphism $i_{M}: F\left(M^{*}\right) \stackrel{\sim}{\longrightarrow}(F M)^{*}$ such that for all $N \in \mathscr{C}$ and $f \in \operatorname{Hom}_{\mathscr{C}}(M, N)$, the following diagram commutes:

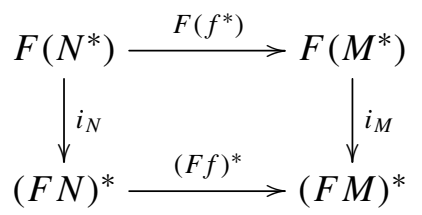

Any duality-preserving functor induces a functor Sesq $(\mathscr{C}) \rightarrow \operatorname{Sesq}\left(\mathscr{C}^{\prime}\right)$, which we also denote by $F$. It is given by

$$
F(M, s)=\left(F M, i_{M} F(s)\right)
$$


for every $(M, s) \in \operatorname{Sesq}(\mathscr{C})$. If the functor $F: \mathscr{C} \rightarrow \mathscr{C}^{\prime}$ is faithful, faithful and full, or induces an equivalence, then the functor $F: \operatorname{Sesq}(\mathscr{C}) \rightarrow \operatorname{Sesq}\left(\mathscr{C}^{\prime}\right)$ shares the same property.

Let $\lambda= \pm 1$. A duality-preserving functor $F$ is called $\lambda$-hermitian if

$$
i_{M^{*}} F\left(\omega_{M}\right)=\lambda i_{M}^{*} \omega_{F M}
$$

for all $M \in \mathscr{C}$. Let $\epsilon= \pm 1$. We recall from [Knus 1991, pp. 80-81] that in this case the functor $F: \operatorname{Sesq}(\mathscr{C}) \rightarrow \operatorname{Sesq}\left(\mathscr{C}^{\prime}\right)$ maps $\mathrm{UH}^{\epsilon}(\mathscr{C})$ to $\mathrm{UH}^{\epsilon \lambda}\left(\mathscr{C}^{\prime}\right)$ and sends $\epsilon$-hermitian hyperbolic forms to $\epsilon \lambda$-hermitian hyperbolic forms. Therefore, $F$ induces a homomorphism between the corresponding Witt groups:

$$
\mathrm{W}^{\epsilon}(F): \mathrm{W}^{\epsilon}(\mathscr{C}) \rightarrow \mathrm{W}^{\epsilon \lambda}\left(\mathfrak{C}^{\prime}\right) .
$$

If $F$ is an equivalence of categories, then $F: \mathrm{UH}^{\epsilon}(\mathscr{C}) \rightarrow \mathrm{UH}^{\epsilon \lambda}\left(\mathscr{C}^{\prime}\right)$ is also an equivalence of categories and the induced group homomorphism $\mathrm{W}^{\epsilon}(F)$ is an isomorphism of groups.

2C. Transfer into the endomorphism ring. The aim of this subsection is to introduce the method of transfer into the endomorphism ring, which allows us to pass from the abstract setting of hermitian categories to that of a ring with involution, which is more concrete. This method will be applied repeatedly in Section 5. Note that it applies well only to reflexive hermitian categories.

Let $\mathscr{C}$ be a reflexive hermitian category, and let $M$ be an object of $\mathscr{C}$, on which we suppose that there exists a unimodular $\epsilon_{0}$-hermitian form $h_{0}$ for a certain $\epsilon_{0}= \pm 1$. Put $E=\operatorname{End}_{\mathscr{C}}(M)$. According to [Quebbemann et al. 1979, Lemma 1.2], the form $\left(M, h_{0}\right)$ induces on $E$ an involution $\sigma$, defined by $\sigma(f)=h_{0}^{-1} f^{*} h_{0}$ for all $f \in E$. Let $\mathscr{P}(E)$ denote the category of projective right $E$-modules of finite type. Then, using $\sigma$, we can consider $\mathscr{P}(E)$ as a reflexive hermitian category (see Example 2.1).

Recall that an idempotent $e \in \operatorname{End}_{\mathscr{C}}(M)$ splits if there exist an object $M^{\prime} \in \mathscr{C}$ and morphisms $i: M^{\prime} \rightarrow M, j: M \rightarrow M^{\prime}$ such that $j i=\operatorname{id}_{M^{\prime}}$ and $i j=e$.

Denote by $\left.\mathscr{C}\right|_{M}$ the full subcategory of $\mathscr{C}$ each object of which is isomorphic to a direct summand of a finite direct sum of copies of $M$. We consider the functor

$$
\mathrm{T}=\mathrm{T}_{\left(M, h_{0}\right)}:=\operatorname{Hom}\left(M,_{-}\right):\left.\mathscr{C}\right|_{M} \rightarrow \mathscr{P}(E)
$$

given by

$$
\begin{aligned}
N & \mapsto \operatorname{Hom}(M, N) \quad \text { for all }\left.N \in \mathscr{C}\right|_{M}, \\
f & \mapsto \mathrm{T}(f) \text { for all } f \in \operatorname{Hom}\left(N, N^{\prime}\right), N,\left.N^{\prime} \in \mathscr{C}\right|_{M},
\end{aligned}
$$

where for all $g \in \operatorname{Hom}(M, N), \mathrm{T}(f)(g)=f g$. In [Quebbemann et al. 1979, Proposition 2.4], it was proved that the functor $\mathrm{T}$ is fully faithful and dualitypreserving with respect to the natural isomorphism $i=\left(i_{N}\right)_{\left.N \in \mathscr{C}\right|_{M}}: \mathrm{T} * \rightarrow * \mathrm{~T}$ given 
by $i_{N}(f)=\mathrm{T}\left(h_{0}^{-1} f^{*} \omega_{N}\right)$ for every $\left.N \in \mathscr{C}\right|_{M}$ and $f \in \operatorname{Hom}\left(M, N^{*}\right)$. In addition, if all the idempotents of $\left.\mathscr{C}\right|_{M}$ split, then $\mathrm{T}$ is an equivalence of categories. By computation, we easily see that $\mathrm{T}$ is $\epsilon_{0}$-hermitian.

Note that for any finite list of (reflexive) objects $M_{1}, \ldots, M_{t} \in \mathscr{C}$ and any $\epsilon_{0}= \pm 1$, there exists a unimodular $\epsilon_{0}$-hermitian form $\left(M, h_{0}\right)$ such that $M_{1}, \ldots,\left.M_{t} \in \mathscr{C}\right|_{M}$. Indeed, let $N=\bigoplus_{i=1}^{t} M_{i}$ and take $\left(M, h_{0}\right)=\left(N \oplus N^{*}, \mathbb{\boxplus}_{N}^{\epsilon_{0}}\right)$. This means that as long as we treat finitely many hermitian forms, we may pass to the context of hermitian forms over rings with involution.

2D. Linear hermitian categories and ring extension. In this subsection we introduce the notion of extension of rings in hermitian categories.

Let $K$ be a commutative ring. Recall that a $K$-category is an additive category $\mathscr{C}$ such that for every $A, B \in \mathscr{C}, \operatorname{Hom}_{\mathscr{C}}(A, B)$ is endowed with a $K$-module structure such that composition is $K$-bilinear. For example, any additive category is in fact a $\mathbb{Z}$-category. An additive covariant functor $F: \mathscr{C} \rightarrow \mathscr{C}^{\prime}$ between two $K$-categories is $K$-linear if the map $F: \operatorname{Hom}_{\mathscr{C}}(A, B) \rightarrow \operatorname{Hom}_{\mathscr{C}^{\prime}}(F A, F B)$ is $K$-linear for all $A, B \in \mathscr{C}$. $K$-linear contravariant functors are defined in the same manner. A $K$-linear hermitian category is a hermitian category $(\mathscr{b}, *, \omega)$ such that $\mathscr{b}$ is a $K$-category and $*$ is $K$-linear.

Fix a commutative ring $K$. Let $\mathscr{C}$ be an additive $K$-category and let $R$ be a $K$-algebra (with unity, not necessarily commutative). We define the extension of the category $\mathscr{C}$ to the ring $R$, denoted $\mathscr{b} \otimes_{K} R$, to be the category whose objects are formal symbols $C \otimes_{K} R$, with $C \in \mathscr{C}$, and whose Hom-sets are defined by

$$
\operatorname{Hom}_{\mathscr{C} \otimes_{K} R}\left(A \otimes_{K} R, B \otimes_{K} R\right)=\operatorname{Hom}_{\mathscr{C}}(A, B) \otimes_{K} R .
$$

The composition in $\mathscr{C} \otimes_{K} R$ is defined in the obvious way. It is straightforward to check that $\mathscr{b} \otimes_{K} R$ is also a $K$-category. Moreover, when $R$ is commutative, $\mathscr{C} \otimes_{K} R$ is an $R$-category. We define the scalar extension functor, $\mathscr{R}_{R / K}: \mathscr{C} \rightarrow \mathscr{C} \otimes_{K} R$ by

$$
\begin{gathered}
\mathscr{R}_{R / K} M=M \otimes_{K} R \quad \text { for all } M \in \mathscr{C}, \\
\mathscr{R}_{R / K} f=f \otimes_{K} 1 \quad \text { for all } f \in \operatorname{Hom}(M, N) .
\end{gathered}
$$

The functor $\mathscr{R}_{R / K}$ is additive and $K$-linear.

In case $K$ is obvious from the context, we write $\mathscr{C}_{R}, M_{R}, f_{R}$ instead of $\mathscr{C}_{K} \otimes_{K} R$, $M \otimes_{K} R, f \otimes_{K} 1$, respectively. (Here, $M \in \mathscr{C}$ and $f$ is a morphism in $\mathscr{b}$.)

Remark 2.2. The scalar extension we have just defined agrees with scalar extension of modules under mild assumptions, but not in general: Let $S$ and $R$ be two $K$ algebras, and write $S_{R}=S \otimes_{K} R$. There is an additive functor $G:(\operatorname{Mod}-S)_{R} \rightarrow$ $\operatorname{Mod}-\left(S_{R}\right)$ given by

$$
G\left(M_{R}\right)=M \otimes_{S} S_{R} \quad \text { and } \quad G(f \otimes a)(m \otimes b)=f m \otimes a b
$$


for all $M, N \in \operatorname{Mod}-S, f \in \operatorname{Hom}_{S}(M, N)$, and $a, b \in R$, and the following diagram commutes:

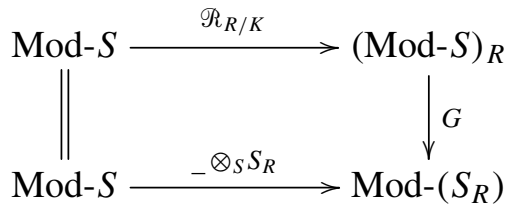

In general, $G$ is neither full nor faithful. However, using standard tensor-Hom relations, it is easy to verify that the map

$$
G: \operatorname{Hom}_{(\operatorname{Mod}-S)_{R}}\left(M_{R}, M_{R}^{\prime}\right) \rightarrow \operatorname{Hom}_{M o d-\left(S_{R}\right)}\left(G M_{R}, G M_{R}^{\prime}\right)
$$

is bijective if either (a) $M$ is finitely generated projective, or (b) $R$ is a flat $K$-module and $M$ is finitely presented. In particular, if $\mathscr{C}$ is an additive subcategory of Mod- $S$ consisting of finitely presented modules and $R$ is flat as a $K$-module, then $\mathscr{C}_{R}$ can be understood as a full subcategory of $\operatorname{Mod}-\left(S_{R}\right)$ in the obvious way. An example in which the map $G$ of (1) is neither injective nor surjective can be obtained by taking $S=K=\mathbb{Z}, R=\mathbb{Q}$ and $M=M^{\prime}=\mathbb{Z}[1 / p] / \mathbb{Z}$.

If $(\mathscr{C}, *, \omega)$ is a $K$-linear hermitian category and $R / K$ is a commutative ring extension, then $\mathscr{C}_{R}$ also has a hermitian structure given by $\left(M_{R}\right)^{*}=\left(M^{*}\right)_{R}$, $(f \otimes a)^{*}=f^{*} \otimes a$ and $\omega_{M_{R}}=\left(\omega_{M}\right)_{R}=\omega_{M} \otimes 1$ for all $M, N \in \mathscr{C}, f \in \operatorname{Hom}_{\mathscr{C}}(M, N)$ and $a \in R$. In this case, the functor $\mathscr{R}_{R / K}$ is a 1-hermitian duality-preserving functor (the natural transformation $i: \mathscr{R}_{R / K} * \rightarrow * \mathscr{R}_{R / K}$ is just the identity). In particular, we get a functor $\mathscr{R}_{R / K}: \operatorname{Sesq}(\mathscr{C}) \rightarrow \operatorname{Sesq}\left(\mathscr{C}_{R}\right)$ given by $\mathscr{R}_{R / K}(M, s):=\left(M_{R}, s_{R}\right)$, and $\mathscr{R}_{R / K}$ sends $\epsilon$-hermitian (hyperbolic) forms to $\epsilon$-hermitian (hyperbolic) forms.

2E. Scalar extension commutes with transfer. Let $R / K$ be a commutative ring extension, let $\mathscr{C}$ be a reflexive $K$-linear hermitian category and let $M$ be an object of $\mathscr{C}$ admitting a unimodular $\epsilon$-hermitian form $h$. Then $\left(M_{R}, h_{R}\right)$ is a unimodular $\epsilon$-hermitian form over $\mathscr{C}_{R}$. Let $E=\operatorname{End}_{\mathscr{C}}(M)$ and $E_{R}=\operatorname{End}_{\mathscr{C}_{R}}\left(M_{R}\right)=E \otimes_{K} R$. It is easy to verify that the following diagram (of functors) commutes:

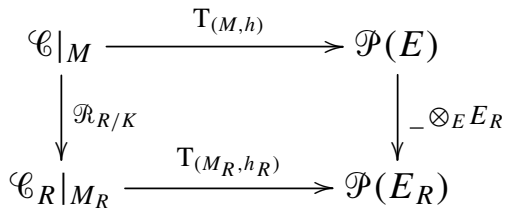

(Note that by Remark 2.2, $\mathscr{P}\left(E_{R}\right)$ and ${ }_{-} \otimes_{E} E_{R}$ can be understood as $\mathscr{P}(E)_{R}$ and $\mathscr{R}_{R / K}$, respectively.) Since all the functors are $\epsilon$ - or 1-hermitian, we get the following commutative diagram, in which the horizontal arrows are full and faithful: 


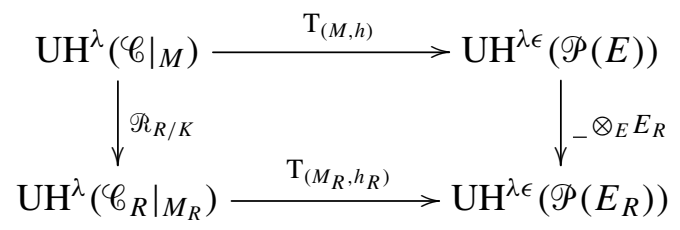

This diagram means that in order to study the behavior of $\mathscr{R}_{R / K}$ on arbitrary $K$ linear hermitian categories, it is enough to study its behavior on hermitian categories obtained from $K$-algebras with $K$-involution (as in Example 2.1).

\section{An equivalence of categories}

Let $\mathscr{C}$ be a (not necessarily reflexive) hermitian category. In this section we prove that there exists a reflexive hermitian category $\mathscr{C}^{\prime}$ such that the category Sesq( $(\mathscr{C})$ is equivalent to $\mathrm{UH}^{1}\left(\mathscr{C}^{\prime}\right)$. (We explain how to extend this result to systems of sesquilinear forms in the next section.)

The category $\mathscr{C}^{\prime}$ resembles the category of double arrows presented in [BayerFluckiger and Moldovan 2014, §3], but is not identical to it. This difference makes our construction work for nonreflexive hermitian categories and, as we shall explain in the next section, for systems of sesquilinear forms, where the forms can be defined with respect to different hermitian structures on $\mathscr{C}$.

3A. The category of twisted double arrows. Let $(\mathscr{C}, *, \omega)$ be a hermitian category. We construct the category of twisted double arrows in $\mathscr{C}$, denoted $\mathrm{A} \tilde{\mathbf{r}}_{2}(\mathscr{C})$, as follows: The objects of $\mathrm{A}_{2}(\mathscr{C})$ are quadruples $(M, N, f, g)$ such that $f, g \in \operatorname{Hom}_{\mathscr{C}}\left(M, N^{*}\right)$. A morphism from $(M, N, f, g)$ to $\left(M^{\prime}, N^{\prime}, f^{\prime}, g^{\prime}\right)$ is a pair $\left(\phi, \psi^{\mathrm{op}}\right)$ such that $\phi \in \operatorname{Hom}\left(M, M^{\prime}\right), \psi \in \operatorname{Hom}\left(N^{\prime}, N\right), f^{\prime} \phi=\psi^{*} f$ and $g^{\prime} \phi=\psi^{*} g$. The composition of two morphisms is given by $\left(\phi, \psi^{\mathrm{op}}\right)\left(\phi^{\prime}, \psi^{\mathrm{op}}\right)=\left(\phi \phi^{\prime},\left(\psi^{\prime} \psi\right)^{\mathrm{op}}\right)$.

The category $\mathrm{A} \tilde{r}_{2}(\mathscr{C})$ is easily seen to be an additive category. Moreover, it has a hermitian structure: For every $(M, N, f, g) \in \mathrm{A} \tilde{\mathbf{r}}_{2}(\mathscr{C})$, define $(M, N, f, g)^{*}=$ $\left(N, M, g^{*} \omega_{N}, f^{*} \omega_{N}\right)$ and $\omega_{(M, N, f, g)}=\operatorname{id}_{(M, N, f, g)}=\left(\operatorname{id}_{M}, \mathrm{id}_{N}^{\mathrm{op}}\right)$. In addition, for every morphism $\left(\phi, \psi^{\mathrm{op}}\right):(M, N, f, g) \rightarrow\left(M^{\prime}, N^{\prime}, f^{\prime}, g^{\prime}\right)$, let $\left(\phi, \psi^{\mathrm{op}}\right)^{*}=\left(\psi, \phi^{\mathrm{op}}\right)$. It is now routine to check that $\left(\mathrm{A} \tilde{\mathbf{r}}_{2}(\mathscr{C}), *, \omega\right)$ is a reflexive hermitian category. Also observe that $* *$ is just the identity functor on $\operatorname{Ar}_{2}(\mathscr{C})$. The following proposition describes the hermitian forms over $\mathrm{A} \tilde{\mathbf{r}}_{2}(\mathscr{C})$ :

Proposition 3.1. Let $Z:=(M, N, f, g) \in \mathrm{A}_{2}(\mathscr{C})$ and let $\alpha, \beta \in \operatorname{Hom}_{\mathscr{C}}(M, N)$. Then $\left(Z,\left(\alpha, \beta^{\mathrm{op}}\right)\right)$ is a hermitian form over $\mathrm{A} \tilde{\mathrm{r}}_{2}(\mathfrak{C})$ if and only if $\alpha=\beta$ and $\alpha^{*} f=g^{*} \omega_{N} \alpha$; equivalently, if and only if $\alpha=\beta$ and $\alpha^{*} g=f^{*} \omega_{N} \alpha$.

Proof. By definition, $Z^{*}=\left(N, M, g^{*} \omega_{N}, f^{*} \omega_{N}\right)$, so $\left(\alpha, \beta^{\text {op }}\right)$ is a morphism from $Z$ to $Z^{*}$ if and only if $\beta^{*} f=g^{*} \omega_{N} \alpha$ and $\beta^{*} g=f^{*} \omega_{N} \alpha$. In addition, by computation, we see $\left(\alpha, \beta^{\text {op }}\right)=\left(\alpha, \beta^{\text {op }}\right)^{*} \circ \omega_{Z}$ precisely when $\alpha=\beta$. Therefore, $\left(Z,\left(\alpha, \beta^{\text {op }}\right)\right)$ is a 
hermitian form if and only if $\alpha=\beta, \alpha^{*} f=g^{*} \omega_{N} \alpha$ and $\alpha^{*} g=f^{*} \omega_{N} \alpha$. It is therefore enough to show $\alpha^{*} f=g^{*} \omega_{N} \alpha$ if and only if $\alpha^{*} g=f^{*} \omega_{N} \alpha$. Indeed, if $\alpha^{*} f=$ $g^{*} \omega_{N} \alpha$, then $\alpha^{*} \omega_{N}^{*} g^{* *}=f^{*} \alpha^{* *}$. Therefore, $\alpha^{*} g=\alpha^{*} \omega_{N}^{*} \omega_{N^{*}} g=\alpha^{*} \omega_{N}^{*} g^{* *} \omega_{M}=$ $f^{*} \alpha^{* *} \omega_{M}=f^{*} \omega_{N} \alpha$, as required (we used the naturality of $\omega$ and the identity $\omega_{N}^{*} \omega_{N^{*}}=\mathrm{id}_{N^{*}}$ in the computation). The other direction follows by symmetry.

Theorem 3.2. Let $\mathscr{C}$ be a hermitian category. Define a functor $F: \operatorname{Sesq}(\mathscr{C}) \rightarrow$ $\mathrm{UH}\left(\mathrm{A} \tilde{r}_{2}(\mathscr{C})\right) b y$

$$
F(M, s)=\left(\left(M, M, s^{*} \omega_{M}, s\right),\left(\mathrm{id}_{M}, \mathrm{id}_{M}^{\mathrm{op}}\right)\right) \quad \text { and } \quad F(\psi)=\left(\psi,\left(\psi^{-1}\right)^{\mathrm{op}}\right)
$$

for all $(M, s) \in \operatorname{Sesq}(\mathscr{C})$ and any morphism $\psi$ in $\operatorname{Sesq}(\mathscr{C})$. Then $F$ induces an equivalence of categories between $\mathrm{Sesq}(\mathscr{C})$ and $\mathrm{UH}\left(\mathrm{A}_{2}(\mathscr{C})\right)$.

Proof. Let $(M, s) \in \operatorname{Sesq}(\mathscr{C})$. That $F(M, s)$ lies in $\mathrm{UH}\left(\mathrm{A}_{2}(\mathscr{C})\right)$ follows from Proposition 3.1. Let $\psi:(M, s) \rightarrow\left(M^{\prime}, s^{\prime}\right)$ be an isometry. Then

$$
\begin{aligned}
F(\psi)^{*}\left(\operatorname{id}_{M^{\prime}}, \operatorname{id}_{M^{\prime}}^{\mathrm{op}}\right) F(\psi) & =\left(\psi,\left(\psi^{-1}\right)^{\mathrm{op}}\right)^{*}\left(\mathrm{id}_{M^{\prime}}, \mathrm{id}_{M^{\prime}}^{\mathrm{op}}\right)\left(\psi,\left(\psi^{-1}\right)^{\mathrm{op}}\right) \\
& =\left(\psi^{-1}, \psi^{\mathrm{op}}\right)\left(\operatorname{id}_{M^{\prime}}, \operatorname{id}_{M^{\prime}}^{\mathrm{op}}\right)\left(\psi,\left(\psi^{-1}\right)^{\mathrm{op}}\right) \\
& =\left(\psi^{-1} \mathrm{id}_{M^{\prime}} \psi,\left(\psi^{-1} \mathrm{id}_{M^{\prime}} \psi\right)^{\mathrm{op}}\right) \\
& =\left(\operatorname{id}_{M}, \mathrm{id}_{M}^{\mathrm{op}}\right)
\end{aligned}
$$

Thus, $F(\psi)$ is an isometry from $F(M, s)$ to $F\left(M^{\prime}, s^{\prime}\right)$. It is clear that $F$ respects composition, so we conclude that $F$ is a functor.

To see that $F$ induces an equivalence, we construct a functor $G$ such that $F$ and $G$ are mutual inverses. Let $G: \mathrm{UH}\left(\mathrm{A}_{2}(\mathscr{C})\right) \rightarrow \operatorname{Sesq}(\mathscr{C})$ be defined by

$$
G\left((M, N, f, g),\left(\alpha, \alpha^{\mathrm{op}}\right)\right)=\left(M, \alpha^{*} g\right) \quad \text { and } \quad G\left(\phi, \psi^{\mathrm{op}}\right)=\phi
$$

for all $\left((M, N, f, g),\left(\alpha, \alpha^{\mathrm{op}}\right)\right) \in \mathrm{UH}\left(\mathrm{Ar}_{2}(\mathscr{C})\right)$ and any morphism $\left(\phi, \psi^{\mathrm{op}}\right)$ in $\mathrm{UH}\left(\tilde{\mathrm{r}}_{2}(\mathscr{C})\right)$.

Let $\left(Z,\left(\alpha, \alpha^{\mathrm{op}}\right)\right),\left(Z^{\prime},\left(\alpha^{\prime}, \alpha^{\prime \mathrm{op}}\right)\right) \in \mathrm{UH}\left(\mathrm{A} \tilde{r}_{2}(\mathscr{C})\right)$ and let $\left(\phi, \psi^{\mathrm{op}}\right)$ be a morphism $\left(Z,\left(\alpha, \alpha^{\mathrm{op}}\right)\right) \rightarrow\left(Z^{\prime},\left(\alpha^{\prime}, \alpha^{\mathrm{op}}\right)\right)$. It is easy to see that $G\left(Z,\left(\alpha, \alpha^{\mathrm{op}}\right)\right)$ lies in Sesq( $\mathscr{C})$, so we now check that $G\left(\phi, \psi^{\mathrm{op}}\right)$ is an isometry from $G\left(Z,\left(\alpha, \alpha^{\mathrm{op}}\right)\right)$ to $G\left(Z^{\prime},\left(\alpha^{\prime}, \alpha^{\prime \text { op }}\right)\right)$. Writing $Z=(M, N, f, g)$ and $Z^{\prime}=\left(M^{\prime}, N^{\prime}, f^{\prime}, g^{\prime}\right)$, this amounts to showing $\alpha^{*} g=\phi^{*} \alpha^{\prime *} g^{\prime} \phi$. Indeed, since $\left(\phi, \psi^{\mathrm{op}}\right)$ is a morphism from $Z$ to $Z^{\prime}$, we have $g^{\prime} \phi=\psi^{*} g$, and since $\left(\phi, \psi^{\text {op }}\right)$ is an isometry, we also have $\left(\phi, \psi^{\mathrm{op}}\right)^{*}\left(\alpha^{\prime}, \alpha^{\mathrm{op}}\right)\left(\phi, \psi^{\mathrm{op}}\right)=\left(\alpha, \alpha^{\mathrm{op}}\right)$, which in turn implies $\psi \alpha^{\prime} \phi=\alpha$. We now have $\phi^{*} \alpha^{*} g^{\prime} \phi=\phi^{*} \alpha^{* *} \psi^{*} g=\left(\psi \alpha^{\prime} \phi\right)^{*} g=\alpha^{*} g$, as required. That $G$ preserves composition is straightforward.

It is easy to see that $G F$ is the identity functor on $\operatorname{Sesq}(\mathscr{C})$, so it is left to show that there is a natural isomorphism from $F G$ to $\operatorname{id}_{\mathrm{UH}\left(\mathrm{Ar}_{2}(\mathscr{C})\right)}$. Keeping the notation 
of the previous paragraph, we have

$$
F G\left((M, N, f, g),\left(\alpha, \alpha^{\mathrm{op}}\right)\right)=\left(\left(M, M,\left(\alpha^{*} g\right)^{*} \omega_{M}, \alpha^{*} g\right),\left(\operatorname{id}_{M}, \mathrm{id}_{M}^{\mathrm{op}}\right)\right) .
$$

By Proposition 3.1 we have $\alpha^{*} f=g^{*} \omega_{N} \alpha$, hence $\left(\alpha^{*} g\right)^{*} \omega_{M}=g^{*} \alpha^{* *} \omega_{M}=$ $g^{*} \omega_{N} \alpha=\alpha^{*} f$. Thus,

$$
F G\left((M, N, f, g),\left(\alpha, \alpha^{\mathrm{op}}\right)\right)=\left(\left(M, M, \alpha^{*} f, \alpha^{*} g\right),\left(\mathrm{id}_{M}, \mathrm{id}_{M}^{\mathrm{op}}\right)\right) .
$$

Define a natural isomorphism $t: \operatorname{id}_{\mathrm{UH}\left(\mathrm{A}_{2}(\mathscr{C})\right)} \rightarrow F G$ by $t_{\left(Z,\left(\alpha, \alpha^{\mathrm{op}}\right)\right)}=\left(\mathrm{id}_{M}, \alpha^{\mathrm{op}}\right)$. Using (2), it is easy to see that $t_{\left(Z,\left(\alpha, \alpha^{\mathrm{op}}\right)\right)}$ is indeed an isometry from $\left(Z,\left(\alpha, \alpha^{\mathrm{op}}\right)\right)$ to $F G\left(Z,\left(\alpha, \alpha^{\mathrm{op}}\right)\right)$. The map $t$ is natural, since for $Z^{\prime},\left(\phi, \psi^{\mathrm{op}}\right)$ as above, we have $F G\left(\phi, \psi^{\mathrm{op}}\right) t_{\left(Z,\left(\alpha, \alpha^{\mathrm{op}}\right)\right)}=\left(\phi,\left(\phi^{-1}\right)^{\mathrm{op}}\right)\left(\mathrm{id}_{M}, \alpha^{\mathrm{op}}\right)=\left(\phi,\left(\alpha \phi^{-1}\right)^{\mathrm{op}}\right)=\left(\phi,\left(\psi \alpha^{\prime}\right)^{\mathrm{op}}\right)=$ $\left(\operatorname{id}_{M^{\prime}}, \alpha^{\prime \mathrm{op}}\right)\left(\phi, \psi^{\mathrm{op}}\right)=t_{\left(Z^{\prime},\left(\alpha^{\prime}, \alpha^{\prime \mathrm{op}}\right)\right)}\left(\phi, \psi^{\mathrm{op}}\right)\left(\right.$ we used the identity $\psi \alpha^{\prime} \phi=\alpha$ verified above).

Remark 3.3. Following [Bayer-Fluckiger and Moldovan 2014, §3], one can also construct the category of (nontwisted) double arrows in $\mathscr{b}$, denoted $\operatorname{Ar}_{2}(\mathscr{C})$. Its objects are quadruples $(M, N, f, g)$ with $M, N \in \mathscr{C}$ and $f, g \in \operatorname{Hom}(M, N)$. A morphism from $(M, N, f, g)$ to $\left(M^{\prime}, N^{\prime}, f^{\prime}, g^{\prime}\right)$ is a pair $(\phi, \psi)$ where $\phi \in$ $\operatorname{Hom}\left(M, M^{\prime}\right)$ and $\psi \in \operatorname{Hom}\left(N, N^{\prime}\right)$ satisfy $\psi f=f^{\prime} \phi$ and $\psi g=g^{\prime} \phi$. The category $\operatorname{Ar}_{2}(\mathscr{C})$ is obviously additive, and, moreover, it admits a hermitian structure given by $(M, N, f, g)^{*}=\left(N^{*}, M^{*}, g^{*}, f^{*}\right),(\phi, \psi)^{*}=\left(\psi^{*}, \phi^{*}\right)$ and $\omega_{(M, N, f, g)}=\left(\omega_{M}, \omega_{N}\right)$.

There is a functor $T: \mathrm{Ar}_{2}(\mathscr{C}) \rightarrow \operatorname{Ar}_{2}(\mathscr{C})$ given by $T(M, N, f, g)=\left(M, N^{*}, f, g\right)$ and $T\left(\phi, \psi^{\mathrm{op}}\right)=\left(\phi, \psi^{*}\right)$. This functor induces an equivalence if $\mathscr{C}$ is reflexive, but otherwise it need neither be faithful nor full. In addition, provided $\mathscr{C}$ is reflexive, one can define a functor $F^{\prime}: \operatorname{Sesq}(\mathscr{C}) \rightarrow \operatorname{UH}\left(\operatorname{Ar}_{2}(\mathscr{C})\right)$ by $F^{\prime}(M, s)=$ $\left(\left(M, M^{*}, s^{*} \omega_{M}, s\right),\left(\omega_{M}, \mathrm{id}_{M^{*}}\right)\right)$ and $F^{\prime}(\psi)=\left(\psi,\left(\psi^{-1}\right)^{*}\right)$. This functor induces an equivalence of categories; the proof is analogous to [Bayer-Fluckiger and Moldovan 2014, Theorem 4.1].

3B. Hyperbolic sesquilinear forms. Let $\mathscr{C}$ be a hermitian category. The equivalence $\operatorname{Sesq}(\mathscr{C}) \sim \mathrm{UH}\left(\mathrm{Ar}_{2}(\mathscr{C})\right)$ of Theorem 3.2 allows us to pull back notions defined for unimodular hermitian forms over $A \tilde{r}_{2}(\mathscr{C})$ to sesquilinear form over $\mathscr{C}$. In this subsection, we will do this for hyperbolicity, and thus obtain a notion of a Witt group of sesquilinear forms.

Throughout, $F$ denotes the functor $\operatorname{Sesq}(\mathscr{C}) \rightarrow \mathrm{UH}\left(\mathrm{A} \tilde{r}_{2}(\mathscr{C})\right)$ from Theorem 3.2.

Definition 3.4. A sesquilinear form $(M, s)$ over $\mathscr{C}$ is called hyperbolic if $F(M, s)$ is hyperbolic as a unimodular hermitian form over $\mathrm{A} \tilde{r}_{2}(\mathscr{C})$.

The following proposition gives a more concrete meaning to hyperbolicity of sesquilinear forms over $\mathfrak{b}$. 
Proposition 3.5. Up to isometry, the hyperbolic sesquilinear forms over $\mathscr{b}$ are given by

$$
\left(M \oplus N,\left[\begin{array}{ll}
0 & f \\
g & 0
\end{array}\right]\right)
$$

where $M, N \in \mathscr{b}, f \in \operatorname{Hom}_{\mathscr{C}}\left(N, M^{*}\right), g \in \operatorname{Hom}_{\mathscr{C}}\left(M, N^{*}\right)$ and $\left[\begin{array}{ll}0 & f \\ g & 0\end{array}\right]$ is an element of $\operatorname{Hom}_{\mathscr{C}}\left(M \oplus N, M^{*} \oplus N^{*}\right)$ given in matrix form. Furthermore, a unimodular $\epsilon$-hermitian form is hyperbolic as a sesquilinear form (i.e., in the sense of Definition 3.4) if and only if it is hyperbolic as a unimodular $\epsilon$-hermitian form (see Section 2).

Proof. Let $G$ be the functor $\operatorname{UH}\left(\mathrm{Ar}_{2}(\mathscr{C})\right) \rightarrow$ Sesq( $\left.\mathscr{C}\right)$ defined in the proof of Theorem 3.2. Since $F$ and $G$ are mutual inverses, the hyperbolic sesquilinear forms over $\mathscr{C}$ are the forms isometric to $G\left(Z \oplus Z^{*}, \mathbb{H}_{Z}\right)$ for $Z \in A \tilde{r}_{2}(\mathscr{C})$. Write $Z=(M, N, h, g)$. Then

$$
\left(Z \oplus Z^{*}, \mathbb{H}_{Z}\right)=\left(\left(M \oplus N, N \oplus M,\left[\begin{array}{cc}
h & 0 \\
0 & g^{*} \omega_{N}
\end{array}\right],\left[\begin{array}{cc}
g & 0 \\
0 & h^{*} \omega_{N}
\end{array}\right]\right),\left[\begin{array}{cc}
0 & \mathrm{id}_{Z^{*}} \\
\omega_{Z} & 0
\end{array}\right]\right) .
$$

Observe that

$$
\left[\begin{array}{cc}
0 & \mathrm{id}_{Z^{*}} \\
\omega_{Z} & 0
\end{array}\right]=\left[\begin{array}{cc}
0 & \left(\mathrm{id}_{N}, \mathrm{id}_{M}^{\mathrm{op}}\right) \\
\left(\mathrm{id}_{M}, \mathrm{id}_{N}^{\mathrm{op}}\right) & 0
\end{array}\right]=\left(\left[\begin{array}{cc}
0 & \mathrm{id}_{N} \\
\mathrm{id}_{M} & 0
\end{array}\right],\left[\begin{array}{cc}
0 & \mathrm{id}_{N} \\
\mathrm{id}_{M} & 0
\end{array}\right]^{\mathrm{op}}\right) .
$$

Thus,

and since

$$
G\left(Z \oplus Z^{*}, \mathbb{H}_{Z}\right)=\left(M \oplus N,\left[\begin{array}{cc}
0 & \operatorname{id}_{N} \\
\operatorname{id}_{M} & 0
\end{array}\right]^{*}\left[\begin{array}{cc}
g & 0 \\
0 & h^{*} \omega_{N}
\end{array}\right]\right),
$$

$$
\left[\begin{array}{cc}
0 & \operatorname{id}_{N} \\
\operatorname{id}_{M} & 0
\end{array}\right]^{*}\left[\begin{array}{cc}
g & 0 \\
0 & h^{*} \omega_{N}
\end{array}\right]=\left[\begin{array}{cc}
0 & \operatorname{id}_{M^{*}} \\
\operatorname{id}_{N^{*}} & 0
\end{array}\right]\left[\begin{array}{cc}
g & 0 \\
0 & h^{*} \omega_{N}
\end{array}\right]=\left[\begin{array}{cc}
0 & h^{*} \omega_{N} \\
g & 0
\end{array}\right],
$$

we see that $G\left(Z \oplus Z^{*}, \mathbb{H}_{Z}\right)$ matches the description in the proposition. Furthermore, by putting $h=f^{*} \omega_{M}$ for $f \in \operatorname{Hom}_{\mathscr{C}}\left(N, M^{*}\right)$, we get $h^{*} \omega_{N}=\omega_{M}^{*} f^{* *} \omega_{N}=$ $\omega_{M}^{*} \omega_{M^{*}} f=f$. Thus, $\left(M \oplus N,\left[\begin{array}{ll}0 & f \\ g & 0\end{array}\right]\right)$ is hyperbolic for all $M, N, f, g$, as required.

To finish, note that we have clearly shown that $\left(Q \oplus Q^{*}, \mathbb{W}_{Q}^{\epsilon}\right)$ is hyperbolic as a sesquilinear form for every $Q \in \mathscr{C}$. To see the converse, assume $\left(M \oplus N,\left[\begin{array}{ll}0 & f \\ g & 0\end{array}\right]\right)$ is $\epsilon$-hermitian and unimodular. Then

$$
\left[\begin{array}{ll}
0 & f \\
g & 0
\end{array}\right]=\epsilon\left[\begin{array}{ll}
0 & f \\
g & 0
\end{array}\right]^{*} \omega_{M \oplus N}=\epsilon\left[\begin{array}{cc}
0 & g^{*} \\
f^{*} & 0
\end{array}\right]\left[\begin{array}{cc}
\omega_{M} & 0 \\
0 & \omega_{N}
\end{array}\right]=\left[\begin{array}{cc}
0 & \epsilon g^{*} \omega_{N} \\
\epsilon f^{*} \omega_{M} & 0
\end{array}\right],
$$

hence $g=\epsilon f^{*} \omega_{N}$ and $f=\epsilon g^{*} \omega_{M}$. Since $\left[\begin{array}{ll}0 & f \\ g & 0\end{array}\right]$ is unimodular, $f$ and $g$ are bijective and hence so are $\omega_{N}$ and $\omega_{M}$. In particular, $M$ is reflexive. It is now routine to verify that the $\operatorname{map~}_{M} \oplus f: M \oplus N \rightarrow M \oplus M^{*}$ is an isometry from $\left(M \oplus N,\left[\begin{array}{ll}0 & f \\ g & 0\end{array}\right]\right)$ to $\left(M \oplus M^{*}, \mathbb{U}_{M}^{\epsilon}\right)$, so the former is hyperbolic in the sense of Section 2. 
Let $(A, \sigma)$ be a ring with involution. When $\mathscr{b}$ is the category of right $A$-modules, considered as a hermitian category as in Example 2.1, we obtain a notion of hyperbolic sesquilinear forms over $(A, \sigma)$. These hyperbolic forms can be characterized as follows:

Proposition 3.6. A sesquilinear form $(M, s)$ over $(A, \sigma)$ is hyperbolic if and only if there are submodules $M_{1}, M_{2} \leq M$ such that $s\left(M_{1}, M_{1}\right)=s\left(M_{2}, M_{2}\right)=0$ and $M=M_{1} \oplus M_{2}$. Furthermore, if $(M, s)$ is unimodular and $\epsilon$-hermitian, then $(M, s)$ is hyperbolic as a sesquilinear space if and only if it is hyperbolic as an $\epsilon$-hermitian unimodular space.

Proof. Recall that for any two right $A$-modules $M_{1}, M_{2}$, we identify $\left(M_{1} \oplus M_{2}\right)^{*}$ with $M_{1}^{*} \oplus M_{2}^{*}$ via $f \leftrightarrow\left(\left.f\right|_{M_{1}},\left.f\right|_{M_{2}}\right)$. Let $(M, s)$ be a sesquilinear space, and assume $M=M_{1} \oplus M_{2}$. By straightforward computation, we see that $s_{r}$ is of the form $\left[\begin{array}{ll}0 & f \\ g & 0\end{array}\right] \in \operatorname{Hom}_{A}\left(M, M^{*}\right)=\operatorname{Hom}_{A}\left(M_{1} \oplus M_{2}, M_{1}^{*} \oplus M_{2}^{*}\right)$ if and only if $s\left(M_{1}, M_{1}\right)=$ $s\left(M_{2}, M_{2}\right)=0$. The proposition therefore follows from Proposition 3.5.

3C. Witt groups of sesquilinear forms. Let $\mathscr{C}$ be a hermitian category. Denote by $\mathrm{WG}_{S}(\mathscr{C})$ the Grothendieck group of isometry classes of sesquilinear forms over $\mathscr{C}$, with respect to orthogonal sum. It is easy to see that the hyperbolic isometry classes span a subgroup of $\mathrm{WG}_{S}(\mathscr{C})$, which we denote by $\mathbb{H}(\mathscr{C})$. The Witt group of sesquilinear forms over $\mathscr{C}$ is defined to the quotient

$$
\mathrm{W}_{S}(\mathscr{C})=\mathrm{WG}_{S}(\mathscr{C}) / \mathbb{M}(\mathscr{C}) .
$$

By definition, we have $\mathrm{W}_{S}(\mathscr{C}) \cong \mathrm{W}\left(\mathrm{Ar}_{2}(\mathscr{C})\right)$. Taking $\mathscr{C}$ to be the category of projective right $A$-modules (or, with a different result, reflexive right $A$-modules, or again arbitrary ones) of finite type and their duals, we obtain a notion of a Witt group for sesquilinear forms over $(A, \sigma)$. Also observe that there is a homomorphism of groups $\mathrm{W}^{\epsilon}(\mathscr{C}) \rightarrow \mathrm{W}_{S}(\mathscr{C})$ given by sending the class of a unimodular $\epsilon$-hermitian form to its corresponding class in $\mathrm{W}_{S}(\mathscr{C})$. Corollary 5.14 below presents sufficient conditions for the injectivity of this homomorphism.

3D. Extension of scalars. Let $R / K$ be a commutative ring extension and let $\mathscr{C}$ be a $K$-linear hermitian category. Then the category $A \tilde{r}_{2}(\mathscr{C})$ is also $K$-linear. For later use, we now check that the scalar extension functor $\mathscr{R}_{R / K}$ of Section $2 \mathrm{D}$ "commutes" with the functor $F$ of Theorem 3.2.

Proposition 3.7. There is a 1-hermitian duality-preserving functor $J: \mathrm{A}_{2}(\mathscr{C})_{R} \rightarrow$ $\mathrm{A} \tilde{\mathbf{r}}_{2}\left(\mathscr{C}_{R}\right)$ making the following diagram commute:

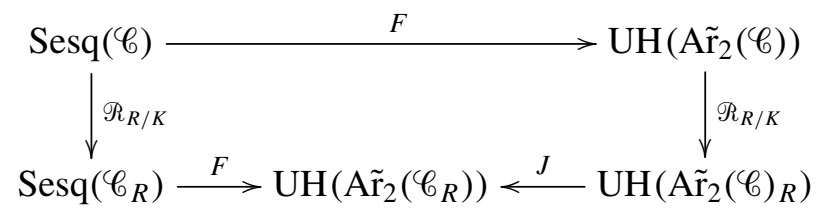


It is given by

$$
\begin{array}{r}
J\left((M, N, f, g)_{R}\right)=\left(M_{R}, N_{R}, f_{R}, g_{R}\right), \\
J\left(\left(\phi, \psi^{\mathrm{op}}\right) \otimes a\right)=\left(\phi \otimes a,(\psi \otimes a)^{\mathrm{op}}\right)
\end{array}
$$

for all $(M, N, f, g) \in \mathrm{A}_{2}(\mathscr{C})$ and any morphism $\left(\phi, \psi^{\mathrm{op}}\right)$ in $\mathrm{A}_{2}(\mathscr{C})$. (The associated natural isomorphism $i: J * \rightarrow * J$ is the identity map.) Furthermore, when $R$ is flat as a $K$-module, $J$ is faithful and full.

Proof. We only check that $J$ is faithful and full when $R$ is flat as a $K$-module. All other assertions follow by computation. Let $Z=(M, N, f, g), Z^{\prime}=\left(M^{\prime}, N^{\prime}, f^{\prime}, g^{\prime}\right)$ be objects in $A \tilde{r}_{2}(\mathscr{C})$. Set

$$
\begin{aligned}
U & =\left\{\left(f, g^{\mathrm{op}}\right) \mid(f, g) \in \operatorname{Hom}_{\mathscr{C}}\left(M, M^{\prime}\right) \times \operatorname{Hom}_{\mathscr{C}}\left(N^{\prime}, N\right)\right\}, \\
V & =\operatorname{Hom}_{\mathscr{C}}\left(M, N^{\prime *}\right) \times \operatorname{Hom}_{\mathscr{C}}\left(M, N^{\prime *}\right),
\end{aligned}
$$

and define $\lambda: U \rightarrow V$ by

$$
\lambda\left(\phi, \psi^{\mathrm{op}}\right)=\left(\psi^{*} f-f^{\prime} \phi, \psi^{*} g-g^{\prime} \phi\right) .
$$

Unfolding the definitions, we see that $\operatorname{Hom}_{\mathrm{Ar}_{2}(\mathscr{\ell})_{R}}\left(Z_{R}, Z_{R}^{\prime}\right)=(\operatorname{ker} \lambda) \otimes_{K} R$ and $\operatorname{Hom}_{\mathrm{Ar}_{2}\left(\mathscr{C}_{R}\right)}\left(J Z_{R}, J Z_{R}^{\prime}\right)=\operatorname{ker}\left(\lambda \otimes_{K} \mathrm{id}_{R}\right)$. Furthermore, the standard map from $(\operatorname{ker} \lambda) \otimes_{K} R$ to $\operatorname{ker}\left(\lambda \otimes_{K} \operatorname{id}_{R}\right)$ is just application of the functor $J$. When $R$ is flat as a $K$-module, this map is an isomorphism; hence we are done.

Corollary 3.8. Let $(M, s),\left(M^{\prime}, s^{\prime}\right)$ be two sesquilinear forms over $\mathscr{C}$, and assume $R$ is flat as a $K$-module. Then $\mathscr{R}_{R / K}(M, s)$ is isometric to $\mathscr{R}_{R / K}\left(M^{\prime}, s^{\prime}\right)$ if and only if $\mathscr{R}_{R / K} F(M, s)$ is isometric to $\mathscr{R}_{R / K} F\left(M^{\prime}, s^{\prime}\right)$.

\section{Systems of sesquilinear forms}

In this section, we explain how to generalize the results of Section 3 to systems of sesquilinear forms.

Let $A$ be a ring, and let $\left\{\sigma_{i}\right\}_{i \in I}$ be a nonempty family of (not necessarily distinct) involutions of $A$. A system of sesquilinear forms over $\left(A,\left\{\sigma_{i}\right\}_{i \in I}\right)$ is a pair $\left(M,\left\{s_{i}\right\}_{i \in I}\right)$ such that $\left(M, s_{i}\right)$ is a sesquilinear space over $\left(A, \sigma_{i}\right)$ for all $i$. An isometry between two systems of sesquilinear forms $\left(M,\left\{s_{i}\right\}_{i \in I}\right),\left(M^{\prime},\left\{s_{i}^{\prime}\right\}_{i \in I}\right)$ is an isomorphism $f: M \rightarrow M^{\prime}$ such that $s_{i}^{\prime}(f x, f y)=s_{i}(x, y)$ for all $x, y \in M$, $i \in I$.

Observe that each of the involutions $\sigma_{i}$ gives rise to a hermitian structure $\left(*_{i}, \omega_{i}\right)$ on Mod- $A$, the category of right $A$-modules. In particular, a system of sesquilinear forms $\left(M,\left\{s_{i}\right\}\right)$ gives rise to homomorphisms $\left(s_{i}\right)_{r},\left(s_{i}\right)_{\ell}: M \rightarrow M^{*_{i}}$ given by $\left(s_{i}\right)_{r}(x)(y)=\sigma_{i}\left(s_{i}(y, x)\right)$ and $\left(s_{i}\right)_{\ell}(x)(y)=s_{i}(x, y)$, where $M^{*_{i}}=\operatorname{Hom}_{A}(M, A)$, considered as a right $A$-module via the action $(f \cdot a) m=\sigma_{i}(a) f(m)$. This leads to the notion of systems of sesquilinear forms over hermitian categories. 
Let $\mathscr{C}$ be an additive category and let $\left\{*_{i}, \omega_{i}\right\}_{i \in I}$ be a nonempty family of hermitian structures on $\mathscr{C}$. A system of sesquilinear forms over $\left(\mathscr{C},\left\{*_{i}, \omega_{i}\right\}_{i \in I}\right)$ is a pair $\left(M,\left\{s_{i}\right\}_{i \in I}\right)$ such that $M \in \mathscr{C}$ and $\left(M, s_{i}\right)$ is a sesquilinear form over $\left(\mathscr{C}, *_{i}, \omega_{i}\right)$. An isometry between two systems of sesquilinear forms $\left(M,\left\{s_{i}\right\}_{i \in I}\right)$ and $\left(M^{\prime},\left\{s_{i}^{\prime}\right\}_{i \in I}\right)$ is an isomorphism $f: M \stackrel{\sim}{\longrightarrow} M^{\prime}$ such that $f^{*_{i}} s_{i}^{\prime} f=s_{i}$ for all $i \in I$. We let $\operatorname{Sesq}_{I}(\mathscr{C})$ (or $\left.\operatorname{Sesq}_{I}\left(\mathscr{C},\left\{*_{i}, \omega_{i}\right\}\right)\right)$ denote the category of systems of sesquilinear forms over $\left(\mathscr{C},\left\{*_{i}, \omega_{i}\right\}_{i \in I}\right)$ with isometries as morphisms.

Keeping the notation of the previous paragraph, the results of Section 3 can be extended to systems of sesquilinear forms as follows: Define the category of twisted double I-arrows over $\left(\mathscr{C},\left\{*_{i}, \omega_{i}\right\}_{i \in I}\right)$, denoted $A \tilde{r}_{2 I}(\mathscr{C})$, to be the category whose objects are quadruples $\left(M, N,\left\{f_{i}\right\}_{i \in I},\left\{g_{i}\right\}_{i \in I}\right)$ with $M, N \in \mathscr{C}$ and $f_{i}, g_{i} \in \operatorname{Hom}_{\mathscr{C}}\left(M, N^{*_{i}}\right)$. A morphism $\left(M, N,\left\{f_{i}\right\},\left\{g_{i}\right\}\right) \rightarrow\left(M^{\prime}, N^{\prime},\left\{f_{i}^{\prime}\right\},\left\{g_{i}^{\prime}\right\}\right)$ is a formal pair $\left(\phi, \psi^{\mathrm{op}}\right)$ such that $\phi \in \operatorname{Hom}\left(M, M^{\prime}\right), \psi \in \operatorname{Hom}\left(N^{\prime}, N\right)$ and $\psi^{*_{i}} f_{i}=f_{i}^{\prime} \phi, \psi^{*_{i}} g_{i}=g_{i}^{\prime} \phi$ for all $i \in I$. The composition is defined by the formula $\left(\phi, \psi^{\mathrm{op}}\right)\left(\phi^{\prime}, \psi^{\mathrm{op}}\right)=\left(\phi \phi^{\prime},\left(\psi^{\prime} \psi\right)^{\mathrm{op}}\right)$.

The category $\mathrm{Ar}_{2 I}(\mathscr{C})$ can be made into a reflexive hermitian category by let$\operatorname{ting}\left(M, N,\left\{f_{i}\right\},\left\{g_{i}\right\}\right)^{*}=\left(N, M,\left\{g_{i}^{*_{i}} \omega_{i, N}\right\},\left\{f_{i}^{* i} \omega_{i, M}\right\}\right),\left(\phi, \psi^{\mathrm{op}}\right)^{*}=\left(\psi, \phi^{\mathrm{op}}\right)$ and $\omega_{\left(M, N,\left\{f_{i}\right\},\left\{g_{i}\right\}\right)}=\left(\mathrm{id}_{M}, \mathrm{id}_{N}^{\mathrm{op}}\right)$. It is now possible to prove the following theorem, whose proof is completely analogous to the proof of Theorem 3.2:

Theorem 4.1. Define a functor $F: \operatorname{Sesq}_{I}(\mathscr{C}) \rightarrow \mathrm{UH}\left(\mathrm{Ar}_{2 I}(\mathscr{C})\right)$ by $F\left(M,\left\{s_{i}\right\}\right)=\left(\left(M, M,\left\{s_{i}^{*_{i}} \omega_{i, M}\right\},\left\{s_{i}\right\}\right),\left(\operatorname{id}_{M}, \operatorname{id}_{M}^{\mathrm{op}}\right)\right)$ and $F(\psi)=\left(\psi,\left(\psi^{-1}\right)^{\mathrm{op}}\right)$.

Then $F$ induces an equivalence of categories.

Sketch of proof. It is easy to see that any hermitian form over $\mathrm{UH}\left(\mathrm{A} \tilde{\mathbf{r}}_{2 I}(\mathscr{C})\right)$ has the form $\left(\left(M, N,\left\{f_{i}\right\},\left\{g_{i}\right\}\right),\left(\alpha, \alpha^{\mathrm{op}}\right)\right)$. Define a functor $G: \mathrm{UH}\left(\mathrm{A}_{2 I}(\mathscr{C})\right) \rightarrow \operatorname{Sesq}_{I}(\mathscr{C})$ by

$$
G\left(\left(M, N,\left\{f_{i}\right\},\left\{g_{i}\right\}\right),\left(\alpha, \alpha^{\mathrm{op}}\right)\right)=\left(M,\left\{\alpha^{*_{i}} g_{i}\right\}\right) \quad \text { and } \quad G\left(\phi, \psi^{\mathrm{op}}\right)=\phi .
$$

Arguing as in the proof of Theorem 3.2, we see that $F$ and $G$ are mutual inverses.

As we did in Section 3, we can use Theorem 4.1 to define hyperbolic systems of sesquilinear forms. Namely, a system of forms $\left(M,\left\{s_{i}\right\}\right)$ over $\mathscr{C}$ will be called hyperbolic if $F\left(M,\left\{s_{i}\right\}\right)$ is hyperbolic over $\mathrm{A} \tilde{\mathbf{r}}_{2 I}(\mathscr{C})$. The following two propositions are proved in the same manner as Propositions 3.5 and 3.6, respectively:

Proposition 4.2. A system of sesquilinear forms $\left(M,\left\{s_{i}\right\}\right)$ over $\mathscr{C}$ is hyperbolic if and only if there are $M_{1}, M_{2} \in \mathscr{C}, f_{i} \in \operatorname{Hom}\left(M_{2}, M_{1}^{*_{i}}\right), g_{i} \in \operatorname{Hom}\left(M_{1}, M_{2}^{*_{i}}\right)$ such that $M=M_{1} \oplus M_{2}$ and, for all $i \in I$,

$$
s_{i}=\left[\begin{array}{cc}
0 & f_{i} \\
g_{i} & 0
\end{array}\right] \in \operatorname{Hom}\left(M, M^{*_{i}}\right)=\operatorname{Hom}\left(M_{1} \oplus M_{2}, M_{1}^{*_{i}} \oplus M_{2}^{*_{i}}\right) .
$$


In this case, each of the sesquilinear forms $\left(M, s_{i}\right)\left(\right.$ over $\left.\left(\mathscr{C}, *_{i}, \omega_{i}\right)\right)$ is hyperbolic.

Proposition 4.3. Let $A$ be a ring and let $\left\{\sigma_{i}\right\}_{i \in I}$ be a nonempty family of involutions of A. A system of sesquilinear forms $\left(M,\left\{s_{i}\right\}\right)$ over $\left(A,\left\{\sigma_{i}\right\}\right)$ is hyperbolic if and only if there are submodules $M_{1}, M_{2} \leq M$ such that $M=M_{1} \oplus M_{2}$ and $s_{i}\left(M_{1}, M_{1}\right)=s_{i}\left(M_{2}, M_{2}\right)=0$ for all $i \in I$. In this case, each of the sesquilinear forms $\left(M, s_{i}\right)$ (over $\left(A, \sigma_{i}\right)$ ) is hyperbolic.

The notion of hyperbolic systems of sesquilinear forms can be used to define Witt groups. We leave the details to the reader.

Let $R / K$ be a commutative ring extension. If $\mathscr{C}$ and all the hermitian structures $\left\{*_{i}, \omega_{i}\right\}_{i \in I}$ are $K$-linear, then the scalar extension functor $\mathscr{R}_{R / K}: \mathscr{C}_{\rightarrow} \rightarrow \mathscr{C}_{R}$ is 1hermitian and duality-preserving with respect to $\left(*_{i}, \omega_{i}\right)$ for all $i \in I$. Therefore, we have a functor $\mathscr{R}_{R / K}: \operatorname{Sesq}_{I}(\mathscr{C}) \rightarrow \operatorname{Sesq}_{I}\left(\mathscr{C}_{R}\right)$ given by $\mathscr{R}_{R / K}\left(M,\left\{s_{i}\right\}_{i \in I}\right)=$ $\left(M_{R},\left\{\left(s_{i}\right)_{R}\right\}_{i \in I}\right)$. We thus have a notion of scalar extension for systems of bilinear forms (and it agrees with the obvious scalar extension for systems of bilinear forms over a ring with a family of involutions, provided the assumptions of Remark 2.2 hold). Using the ideas of Section 3D, one can show:

Corollary 4.4. Let $\left(M,\left\{s_{i}\right\}\right),\left(M^{\prime},\left\{s_{i}^{\prime}\right\}\right)$ be two systems of sesquilinear forms over $\left(\mathscr{C},\left\{*_{i}, \omega_{i}\right\}\right)$, and assume $R$ is flat as a $K$-module. Then $\mathscr{R}_{R / K}\left(M,\left\{s_{i}\right\}\right)$ is isometric to $\mathscr{R}_{R / K}\left(M^{\prime},\left\{s_{i}^{\prime}\right\}\right)$ if and only if $\mathscr{R}_{R / K} F\left(M,\left\{s_{i}\right\}\right)$ is isometric to $\mathscr{R}_{R / K} F\left(M^{\prime},\left\{s_{i}^{\prime}\right\}\right)$.

\section{Applications}

This section uses the previous results to generalize various known results about hermitian forms (over rings or reflexive hermitian categories) to systems of sesquilinear forms over (not necessarily reflexive) hermitian categories. Some of the consequences to follow were obtained in [Bayer-Fluckiger and Moldovan 2014] for hermitian forms over rings. Here we rephrase them for hermitian categories, extend them to systems of sesquilinear forms and drop the assumption that the base module (or object) is reflexive.

5A. Witt's cancellation theorem. Quebbemann, Scharlau and Schulte [Quebbemann et al. 1979, §3.4] proved Witt's cancellation theorem for unimodular hermitian forms over hermitian categories $\mathscr{C}$ satisfying the following conditions:

(a) All idempotents in $\mathscr{C}$ split (see Section 2C).

(b) For all $C \in \mathscr{C}, E:=\operatorname{End}_{\mathscr{C}}(C)$ is a complete semilocal ring in which 2 is invertible.

Recall that complete semilocal means that $E / \operatorname{Jac}(E)$ is semisimple (i.e., $E$ is semilocal) and that the standard map $E \rightarrow \lim \left\{E / \operatorname{Jac}(R)^{n}\right\}_{n \in \mathbb{N}}$ is an isomorphism (i.e., $E$ is complete in the $\operatorname{Jac}(E)$-adic topology). In fact, condition (a) can be 
dropped since idempotents can be split artificially (see Section 5E below), or, alternatively, since by applying transfer (see Section 2C) one can move to a module category in which idempotents split.

We shall now use the Quebbemann-Scharlau-Schulte cancellation theorem together with Theorem 4.1 to give several conditions guaranteeing cancellation for systems of sesquilinear forms.

Our first criterion is based on the following well-known lemma:

Lemma 5.1. Let $K$ be a commutative noetherian complete semilocal ring (e.g., a complete discrete valuation ring). Then any $K$-algebra $A$ which is finitely generated as a $\mathrm{K}$-module is complete semilocal.

Proof. For brevity, write $I=\operatorname{Jac}(K)$ and $J=\operatorname{Jac}(A)$. By [Hinohara 1960, Theorem 2] and the proof of [First 2013, Proposition 8.8(i)] (for instance), $A=$ $\lim \left\{A / A\left(I^{n}\right)\right\}_{n \in \mathbb{N}}$. That $A=\lim \left\{A / J^{n}\right\}_{n \in \mathbb{N}}$ follows if we verify that $J^{m} \subseteq A I \subseteq J$ for some $m \in \mathbb{N}$. The right inclusion holds since $1+A I$ consists of right-invertible elements. Indeed, for all $a \in A I$, we have $a A+A I=A$, so by Nakayama's lemma (applied to the $K$-module $A$ ), $a A=A$. The existence of $m$, as well as the fact that $A$ is semilocal, follows by arguing as in [Rowen 1988, Example 2.7.19'(ii)] (for instance).

Theorem 5.2. Let $K$ be a commutative noetherian complete semilocal ring with $2 \in$ $K^{\times}$, let $\mathscr{C}$ be a $K$-category equipped with $K$-linear hermitian structures $\left\{*_{i}, \omega_{i}\right\}_{i \in I}$, and let $\left(M,\left\{s_{i}\right\}\right),\left(M^{\prime},\left\{s_{i}^{\prime}\right\}\right),\left(M^{\prime \prime},\left\{s_{i}^{\prime \prime}\right\}\right)$ be systems of sesquilinear forms over $\left(\mathscr{b},\left\{*_{i}, \omega_{i}\right\}\right)$. Assume that $\operatorname{Hom}_{\mathscr{C}}(M, N)$ is finitely generated as a $K$-module for all $M, N \in \mathscr{C}$. Then

$$
\left(M,\left\{s_{i}\right\}\right) \oplus\left(M^{\prime},\left\{s_{i}^{\prime}\right\}\right) \simeq\left(M,\left\{s_{i}\right\}\right) \oplus\left(M^{\prime \prime},\left\{s_{i}^{\prime \prime}\right\}\right) \Longleftrightarrow\left(M^{\prime},\left\{s_{i}^{\prime}\right\}\right) \simeq\left(M^{\prime \prime},\left\{s_{i}^{\prime \prime}\right\}\right) .
$$

Proof. In light of Theorem 4.1, it is enough to prove cancellation of unimodular 1hermitian forms over the category $\mathrm{Ar}_{2 I}(\mathscr{C})$ (note that the equivalence of Theorem 4.1 respects orthogonal sums). This would follow from the cancellation theorem of [Quebbemann et al. 1979, §3.4] if we show that the endomorphism rings of objects in $A \tilde{\mathbf{r}}_{2 I}(\mathscr{C})$ are complete semilocal rings in which 2 is invertible. Indeed, let $Z:=\left(M, N,\left\{f_{i}\right\},\left\{g_{i}\right\}\right) \in \operatorname{Ar}_{2 I}(\mathscr{C})$. Then $E:=\operatorname{End}(Z)$ is a subring of $\operatorname{End}_{\mathscr{C}}(M) \times$ $\operatorname{End}_{\mathscr{C}}(N)^{\text {op }}$, which is a $K$-algebra by assumption. Since the hermitian structures $\left\{*_{i}, \omega_{i}\right\}$ are $K$-linear, $E$ is in fact a $K$-subalgebra, which must be finitely generated as a $K$-module (because this is true for $\operatorname{End}_{\mathscr{C}}(M) \times \operatorname{End}_{\mathscr{C}}(N)^{\text {op }}$ and $K$ is noetherian). Thus, we are done by Lemma 5.1 and the fact that $2 \in K^{\times}$.

As corollary, we get the following result, which resembles [Bayer-Fluckiger and Moldovan 2014, Theorem 8.1]:

Corollary 5.3. Let $K$ be a commutative noetherian complete semilocal ring with $2 \in K^{\times}$, let $A$ be a $K$-algebra which is finitely generated as a $K$-module, and let 
$\left\{\sigma_{i}\right\}_{i \in I}$ be a family of $K$-involutions on A. Then cancellation holds for systems of sesquilinear forms over $\left(A,\left\{\sigma_{i}\right\}\right)$ which are defined on finitely generated right A-modules.

For the next theorem, recall that a ring $R$ is said to be semiprimary if $R$ is semilocal and $\operatorname{Jac}(R)$ is nilpotent. For example, all artinian rings are semiprimary. Note that all semiprimary rings are complete semilocal. It is well-known that for a ring $R$ and an idempotent $e \in R, R$ is semiprimary if and only if $e R e$ and $(1-e) R(1-e)$ are semiprimary. As a result, if $M, N$ are two objects in an additive category, then $\operatorname{End}(M \oplus N)$ is semiprimary if and only if $\operatorname{End}(M)$ and $\operatorname{End}(N)$ are semiprimary.

Theorem 5.4. Let $\mathscr{C}$ be an additive category with hermitian structures $\left\{*_{i}, \omega_{i}\right\}$, and let $\left(M,\left\{s_{i}\right\}\right),\left(M^{\prime},\left\{s_{i}^{\prime}\right\}\right),\left(M^{\prime \prime},\left\{s_{i}^{\prime \prime}\right\}\right)$ be systems of sesquilinear forms over $\left(\mathscr{C},\left\{*_{i}, \omega_{i}\right\}\right)$. Assume that $\operatorname{End}_{\mathscr{C}}(M), \operatorname{End}_{\mathscr{C}}\left(M^{\prime}\right), \operatorname{End}_{\mathscr{C}}\left(M^{\prime \prime}\right)$ are semiprimary rings in which 2 is invertible. Then

$$
\left(M,\left\{s_{i}\right\}\right) \oplus\left(M^{\prime},\left\{s_{i}^{\prime}\right\}\right) \simeq\left(M,\left\{s_{i}\right\}\right) \oplus\left(M^{\prime \prime},\left\{s_{i}^{\prime \prime}\right\}\right) \Longleftrightarrow\left(M^{\prime},\left\{s_{i}^{\prime}\right\}\right) \simeq\left(M^{\prime \prime},\left\{s_{i}^{\prime \prime}\right\}\right) .
$$

Proof. As in the proof of Theorem 5.2, it is enough to show that the objects in A $\tilde{\mathbf{r}}_{2 I}(\mathscr{C})$ have a complete semilocal endomorphism ring. In fact, we may restrict to those objects $Z:=\left(M, N,\left\{f_{i}\right\},\left\{g_{i}\right\}\right)$ for which $\operatorname{End}_{\mathscr{C}}(M)$ and $\operatorname{End}_{\mathscr{C}}(N)$ are semiprimary. (These do form a hermitian subcategory of $A \tilde{r}_{2 I}(\mathscr{C})$ by the comments above.) Fix such a $Z$ and let $H=\bigoplus_{i \in I} \operatorname{Hom}_{\mathscr{C}}\left(M, N^{*_{i}}\right)$. We view the morphism $\left\{f_{i}\right\}$ and $\left\{g_{i}\right\}$ as elements of $H$ in the obvious way. Let $A=\operatorname{End}(M)$ and $B=\operatorname{End}(N)$. We endow $H$ with a $\left(B^{\mathrm{op}}, A\right)$-bimodule structure by setting $b^{\mathrm{op}}\left(\bigoplus_{i \in I} h_{i}\right) a=$ $\bigoplus_{i \in I}\left(b^{*_{i}} \circ h_{i} \circ a\right)$ for all $a \in A, b \in B, \bigoplus_{i} h_{i} \in H$. This allows us to construct the ring $S:=\left[\begin{array}{ll}A & \\ H & B^{\text {op }}\end{array}\right]$. It is now straightforward to check that $\operatorname{End}(Z)$ consists of those elements in $A \times B^{\mathrm{op}}=\left[\begin{array}{cc}A & \\ & B^{\text {op }}\end{array}\right]$ that commute with $\left[\begin{array}{c}0 \\ f_{i} 0\end{array}\right]$ and $\left[\begin{array}{c}0 \\ g_{i} 0\end{array}\right]$ for all $i \in I$. Thus, $\operatorname{End}(Z)$ is a semicentralizer subring of $A \times B^{\mathrm{oP}}$ in the sense of [First 2013, $\S 1$ ]. By [First 2013, Theorem 4.6], a semicentralizer subring of a semiprimary ring is semiprimary, so $\operatorname{End}(Z)$ is semiprimary, and in particular complete semilocal.

Corollary 5.5. Let $A$ be a semiprimary ring with $2 \in A^{\times}$, and let $\left\{\sigma_{i}\right\}_{i \in I}$ be a family of involutions on $A$. Then cancellation holds for systems of sesquilinear forms over $\left(A,\left\{\sigma_{i}\right\}\right)$ which are defined on finitely presented right A-modules.

Proof. By [Björk 1971, Theorem 4.1] (or [First 2013, Theorem 7.3]), the endomorphism ring of a finitely presented $A$-module is semiprimary. Now apply Theorem 5.4.

Corollary 5.6. Let $\mathscr{C}$ be an abelian category equipped with hermitian structures $\left\{*_{i}, \omega_{i}\right\}$. Assume that $\mathscr{C}$ consists of objects of finite length. Then cancellation holds for systems of sesquilinear forms over $\left(\mathscr{C},\left\{*_{i}, \omega_{i}\right\}\right)$. 
Proof. By the Harada-Sai lemma [Rowen 1988, Proposition 2.9.29], the endomorphism ring of an object of finite length in an abelian category is semiprimary, so we are done by Theorem 5.4. Alternatively, one can check directly that the category $\mathrm{A} \tilde{r}_{2 I}(\mathscr{C})$ is abelian and consists of objects of finite length, apply the Harada-Sai lemma to $A \tilde{r}_{2 I}(\mathscr{C})$, and then use the cancellation theorem of [Quebbemann et al. 1979, §3.4].

Remark 5.7. It is not hard to deduce from a theorem of Camps and Dicks [1993, Corollary 2] that if the endomorphism rings of $\mathscr{C}$ are semilocal, then so are the endomorphism rings of $A \tilde{\mathbf{r}}_{2 I}(\mathscr{C})$. (Simply check that $\operatorname{End}\left(M, N,\left\{f_{i}\right\},\left\{g_{i}\right\}\right)$ is a rationally closed subring of $\operatorname{End}_{\mathscr{C}}(M) \times \operatorname{End}_{\mathscr{C}}(N)^{\mathrm{op}}$ in the sense of [Camps and Dicks 1993, p. 204].) By applying transfer (see Section 2C) to A $\tilde{\mathbf{r}}_{2 I}(\mathscr{C})$, one can then move to the context of unimodular 1-hermitian forms over semilocal rings. Cancellation theorems for such forms were obtained by various authors, including Knebusch [1969], Reiter [1975] and Keller [1988]. However, none of these apply to the general case, as in fact cancellation is no longer true; see [Keller 1988, §2]. Nevertheless, the cancellation results of [ibid.] can still be used to get some partial results about systems of sesquilinear forms over $\mathscr{b}$; we leave the details to the reader.

5B. Finiteness results. In this subsection and the next, we generalize the finiteness results of [Bayer-Fluckiger and Moldovan 2014, §10] to systems of sesquilinear forms.

For a ring $A$, we denote by $T(A)$ the $\mathbb{Z}$-torsion subgroup of $A$. Recall that if $R$ is a commutative ring, $A$ is said to be $R$-finite if $A_{R}=A \otimes_{\mathbb{Z}} R$ is a finitely generated $R$-module and $T(A)$ is finite. Note that being $R$-finite passes to subrings.

The proofs of the results to follow are completely analogous to the proofs of the corresponding statements in [Bayer-Fluckiger and Moldovan 2014, §10]; they are based on applying the equivalence of Theorem 4.1 and then using the finiteness results of [Bayer-Fluckiger et al. 1989], possibly after applying transfer.

Throughout, $\mathscr{C}$ is an additive category and $\left\{*_{i}, \omega_{i}\right\}_{i \in I}$ is a nonempty family of hermitian structures on $\mathscr{C}$. Fix a system of sesquilinear forms $\left(V,\left\{s_{i}\right\}_{i \in I}\right)$ over $\left(\mathscr{C},\left\{{ }_{i}, \omega_{i}\right\}\right)$ and let $Z\left(V,\left\{s_{i}\right\}\right)=\left(V, V,\left\{s_{i}^{*} \omega_{i, V}\right\},\left\{s_{i r}\right\}\right) \in \mathrm{A}_{2 I}(\mathscr{C})$. (Note that $F\left(V,\left\{s_{i}\right\}\right)=\left(Z,\left(\operatorname{id}_{V}, \mathrm{id}_{V}^{\mathrm{op}}\right)\right)$ with $F$ as in Theorem 4.1.)

Theorem 5.8. If there exists a nonzero integer $m$ such that $\operatorname{End}_{\mathscr{C}}(V)$ is $\mathbb{Z}[1 / m]$ finite, then there are finitely many isometry classes of summands of $\left(V,\left\{s_{i}\right\}\right)$.

Theorem 5.9. Assume that there exists a nonzero integer $m$ such that the ring $\operatorname{End}_{\mathrm{Ar}_{2 I}(\mathscr{C})}\left(Z\left(V,\left\{s_{i}\right\}\right)\right)$ is $\mathbb{Z}[1 / m]$-finite $\left(\right.$ e.g., if $\operatorname{End}_{\mathscr{C}}(V)$ is $\mathbb{Z}[1 / m]$-finite). Then there exist only finitely many isometry classes of systems of sesquilinear forms $\left(V^{\prime},\left\{s_{i}^{\prime}\right\}_{i \in I}\right)$ over $\mathscr{C}$ such that $Z\left(V^{\prime},\left\{s_{i}^{\prime}\right\}\right) \simeq Z\left(V,\left\{s_{i}\right\}\right)$ (as objects in $\left.\mathrm{A}_{2 I}(\mathfrak{C})\right)$. 
5C. Finiteness of the genus. Let $\mathscr{C}$ be a hermitian category admitting a nonempty family of hermitian structures $\left\{*_{i}, \omega_{i}\right\}_{i \in I}$. We say that two systems of sesquilinear forms $\left(M,\left\{s_{i}\right\}\right),\left(M^{\prime},\left\{s_{i}^{\prime}\right\}\right)$ are of the same genus if they become isometric after applying $\mathscr{R}_{\mathbb{Z}_{p} / \mathbb{Z}}$ for every prime number $p$ (where $\mathbb{Z}_{p}$ are the $p$-adic integer). (See Remark 2.2 for conditions under which this definition of genus agrees with the naive definition of genus for module categories.) As in [Bayer-Fluckiger and Moldovan 2014, Theorem 10.3], we have:

Theorem 5.10. Let $\left(M,\left\{s_{i}\right\}\right)$ be a system of sesquilinear forms over $\left(\mathscr{C},\left\{*_{i}, \omega_{i}\right\}\right)$, and assume that $\operatorname{End}(M)$ is $\mathbb{Q}$-finite. Then the genus of $\left(M,\left\{s_{i}\right\}\right)$ contains only a finite number of isometry classes of systems of sesquilinear forms.

5D. Forms that are trivial in the Witt group. Let $\mathscr{C}$ be a hermitian category. By definition, a unimodular $\epsilon$-hermitian (resp. sesquilinear) form $(M, s)$ is trivial in $\mathrm{W}^{\epsilon}(\mathscr{C})$ (resp. $\mathrm{W}_{S}(\mathscr{C})$ ) if and only if there are unimodular $\epsilon$-hermitian (resp. sesquilinear) hyperbolic forms $\left(H_{1}, h_{1}\right),\left(H_{2}, h_{2}\right)$ such that $(M, s) \oplus\left(H_{1}, h_{1}\right) \simeq$ $\left(H_{2}, h_{2}\right)$. In this section, we will show that under mild assumptions, this implies that $(M, s)$ is hyperbolic.

Lemma 5.11. Let $M \in \mathscr{C}$, and assume that $M$ is a (finite) direct sum of objects with local endomorphism ring. Then, up to isometry, there is at most one $\epsilon$-hermitian hyperbolic form on $M$.

Proof. For $X \in \mathscr{C}$, let $[X]$ denote the isomorphism class of $X$. The Krull-Schmidt theorem (e.g., see [Rowen 1988, p. 237 ff.]) implies that if $M \cong \bigoplus_{i=1}^{t} M_{i}$ with each $M_{i}$ indecomposable, then the unordered list $\left[M_{1}\right], \ldots,\left[M_{t}\right]$ is determined by $M$.

Let $(M, s)$ be an $\epsilon$-hermitian hyperbolic form on $M$, say $(M, s) \simeq\left(N \oplus N^{*}, \mathbb{H} \mathbb{H}_{N}^{\epsilon}\right)$. Write $N \cong \bigoplus_{i=1}^{r} N_{i}$ with each $N_{i}$ indecomposable. Then $s \simeq \bigoplus_{i=1}^{r} \mathbb{E}_{N_{i}}^{\epsilon}$. It is easy to check that the isometry class of $\mathbb{H}_{N_{i}}^{\epsilon}$ depends only on the set $\left\{\left[N_{i}\right],\left[N_{i}^{*}\right]\right\}$. Furthermore, using the Krull-Schmidt theorem, one easily verifies that the unordered list $\left\{\left[N_{1}\right],\left[N_{1}^{*}\right]\right\}, \ldots,\left\{\left[N_{r}\right],\left[N_{r}^{*}\right]\right\}$ is uniquely determined by $M$. It follows that $(M, s)$ is isometric to a sesquilinear form which is determined by $M$ up to isometry.

Proposition 5.12. Let $\mathscr{C}$ be a hermitian category satisfying conditions (a), (b) on page 15. Then a unimodular $\epsilon$-hermitian form $(M, s)$ is trivial in $\mathrm{W}^{\epsilon}(\mathscr{C})$ if and only if it is hyperbolic.

Proof. Note first that conditions (a) and (b) imply that every object of $\mathscr{C}$ is a sum of objects with local endomorphism rings, hence we may apply the Krull-Schmidt theorem to $\mathscr{C}$. (For example, this follows from [Rowen 1988, Theorem 2.8.40] since the endomorphism rings of $\mathscr{C}$ are semiperfect.) Let $(M, s)$ be a unimodular $\epsilon$-hermitian form such that $(M, s) \equiv 0$ in $\mathrm{W}^{\epsilon}(\mathscr{C})$. There are unimodular $\epsilon$-hermitian hyperbolic forms $\left(H_{1}, h_{1}\right),\left(H_{2}, h_{2}\right)$ such that $(M, s) \oplus\left(H_{1}, h_{1}\right) \simeq\left(H_{2}, h_{2}\right)$. Using 
the Krull-Schmidt theorem, it is easy to see that there is $N \in \mathscr{C}$ such that $M \cong N \oplus N^{*}$. Thus, we may consider $\mathbb{U}_{N}^{\epsilon}$ as a hermitian form on $M$. By Lemma 5.11, we have $\mathbb{H}_{N}^{\epsilon} \oplus h_{1} \simeq h_{2}$, implying $\mathbb{H}_{N}^{\epsilon} \oplus h_{2} \simeq s \oplus h_{2}$. Therefore, by the cancellation theorem of [Quebbemann et al. 1979, §3.4], $s \simeq \mathbb{I}_{N}^{\epsilon}$, as required.

Proposition 5.13. Let $\mathscr{C}$ be a hermitian category in which all idempotents split and such that either

(1) $\mathscr{C}$ is $K$-linear, where $K$ is a noetherian complete semilocal ring with $2 \in K^{\times}$, and all Hom-sets in $\mathscr{C}$ are finitely generated as $K$-modules, or

(2) for all $M \in \mathscr{C}, \operatorname{End}_{\mathscr{C}}(M)$ is semiprimary and $2 \in \operatorname{End}_{\mathscr{C}}(M)^{\times}$.

Then a sesquilinear form $(M, s)$ is trivial in $\mathrm{W}_{S}(\mathscr{C})$ if and only if it is hyperbolic.

Proof. It is enough to verify that $F(M, s)$ is hyperbolic in $\mathrm{Ar}_{2}(\mathscr{C})$ (Theorem 3.2). The proofs of Theorems 5.2 and 5.4 imply that $A \tilde{r}_{2}(\mathscr{C})$ satisfies condition (b) of Section 5A, and condition (a) is routine (see also Lemma 5.17(ii) below). Therefore, $F(M, s)$ is hyperbolic by Proposition 5.12.

Corollary 5.14. Under the assumptions of Proposition 5.13, the map $\mathrm{W}(\mathscr{C}) \rightarrow$ $\mathrm{W}_{S}(\mathscr{C})$ is injective.

Proof. This follows from Propositions 5.13 and 3.5.

5E. Odd degree extensions. Throughout this subsection, $L / K$ is an odd degree field extension and char $K \neq 2$. A well-known theorem of Springer asserts that two unimodular hermitian forms over $K$ become isometric over $L$ if and only if they are already isometric over $K$. Moreover, the restriction map (the scalar extension map) $r_{L / K}: \mathrm{W}(K) \rightarrow \mathrm{W}(L)$ is injective. Both statements were extended to hermitian forms over finite-dimensional $K$-algebras with $K$-linear involution in [Bayer-Fluckiger and Lenstra 1990, Proposition 1.2 and Theorem 2.1] (see also [Fainsilber 1994] for a version in which $L / K$ is replaced with an extension of complete discrete valuation rings). In this section, we extend these results to sesquilinear forms over hermitian categories.

Theorem 5.15. Let $\mathscr{C}$ be an additive $K$-category such that $\operatorname{dim}_{K} \operatorname{Hom}\left(M, M^{\prime}\right)$ is finite for all $M, M^{\prime} \in \mathscr{C}$. Let $\left\{*_{i}, \omega_{i}\right\}_{i \in I}$ be a nonempty family of $K$-linear hermitian structures on $\mathscr{C}$ and let $\left(M,\left\{s_{i}\right\}\right),\left(M^{\prime},\left\{s_{i}^{\prime}\right\}\right)$ be two systems of sesquilinear forms over $\left(\mathscr{C},\left\{*_{i}, \omega_{i}\right\}\right)$. Then $\mathscr{R}_{L / K}\left(M,\left\{s_{i}\right\}\right) \simeq \mathscr{R}_{L / K}\left(M^{\prime},\left\{s_{i}^{\prime}\right\}\right)$ if and only if $\left(M,\left\{s_{i}\right\}\right) \simeq\left(M^{\prime},\left\{s_{i}^{\prime}\right\}\right)$.

Proof. By Corollary 4.4, it is enough to prove $\mathscr{R}_{L / K} F\left(M,\left\{s_{i}\right\}\right) \simeq \mathscr{R}_{L / K} F\left(M^{\prime},\left\{s_{i}^{\prime}\right\}\right)$ if and only if $F\left(M,\left\{s_{i}\right\}\right) \simeq F\left(M^{\prime},\left\{s_{i}^{\prime}\right\}\right)$ (with $F$ as in Theorem 4.1). Write $\left(Z,\left(\alpha, \alpha^{\mathrm{op}}\right)\right)=F\left(M,\left\{s_{i}\right\}\right) \oplus F\left(M^{\prime},\left\{s_{i}^{\prime}\right\}\right)$ and let $E=\operatorname{End}(Z)$. Then $E$ is a $K$ subalgebra of $\operatorname{End}\left(M \oplus M^{\prime}\right) \times \operatorname{End}\left(M \oplus M^{\prime}\right)^{\text {op }}$, which is finite-dimensional. By applying $\mathrm{T}_{\left(Z,\left(\alpha, \alpha^{\mathrm{op}}\right)\right)}$ (see Section $2 \mathrm{C}$ ), we reduce to showing that two 1-hermitian 
forms over $E$ are isometric over $E \otimes_{K} L$ if and only if they are isometric over $E$, which is just [Bayer-Fluckiger and Lenstra 1990, Theorem 2.1]. (Note that we used the fact that transfer commutes with $\mathscr{R}_{L / K}$ in the sense of Section 2E.)

Corollary 5.16. Let $A$ be a finite-dimensional $K$-algebra and let $\left\{\sigma_{i}\right\}_{i \in I}$ be a nonempty family of $K$-involutions on $A$. Let $\left(M,\left\{s_{i}\right\}\right),\left(M^{\prime},\left\{s_{i}^{\prime}\right\}\right)$ be two systems of sesquilinear forms over $\left(A,\left\{\sigma_{i}\right\}\right)$. If $M$ and $M^{\prime}$ are of finite type, then $\mathscr{R}_{L / K}\left(M,\left\{s_{i}\right\}\right) \simeq \mathscr{R}_{L / K}\left(M^{\prime},\left\{s_{i}^{\prime}\right\}\right)$ if and only if $\left(M,\left\{s_{i}\right\}\right) \simeq\left(M^{\prime},\left\{s_{i}^{\prime}\right\}\right)$.

To state the analogue of the injectivity of $r_{L / K}: \mathrm{W}(K) \rightarrow \mathrm{W}(L)$ for hermitian categories, we need to introduce additional notation.

An additive category $\mathscr{C}$ is called pseudoabelian if all idempotents in $\mathscr{C}$ split. Any additive category $\mathscr{C}$ admits a pseudoabelian closure (e.g., see [Karoubi 1978, Theorem 6.10]), namely, a pseudoabelian additive category $\mathscr{C}^{\circ}$ equipped with an additive functor $A \mapsto A^{\circ}: \mathscr{C} \rightarrow \mathscr{C}^{\circ}$, such that the pair $\left(\mathscr{C}^{\circ}, A \mapsto A^{\circ}\right)$ is universal. The category $\mathscr{C}^{\circ}$ is unique up to equivalence and the functor $A \mapsto A^{\circ}$ turns out to be faithful and full. The category $\mathscr{C}^{\circ}$ can be realized as the category of pairs $(M, e)$ with $M \in \mathscr{C}$ and $e \in \operatorname{End}_{\mathscr{C}}(M)$ an idempotent. The Hom-sets in $\mathscr{C}^{\circ}$ are given by $\operatorname{Hom}_{\mathscr{C}} \circ\left((M, e),\left(M^{\prime}, e^{\prime}\right)\right)=e^{\prime} \operatorname{Hom}_{\mathscr{C}}\left(M, M^{\prime}\right) e$ and the composition is the same as in $\mathscr{C}$. Finally, set $M^{\circ}=\left(M, \mathrm{id}_{M}\right)$ and $f^{\circ}=f$ for any object $M \in \mathscr{C}$ and any morphism $f$ in $\mathscr{C}$. For simplicity, we will use only this particular realization of $\mathscr{C}^{\circ}$. Nevertheless, the universality implies that the statements to follow hold for any pseudoabelian closure.

Assume $\mathscr{C}$ admits a $K$-linear hermitian structure $(*, \omega)$. Then $\mathscr{C}^{\circ}$ is clearly a $K$-category, and, moreover, has a $K$-linear hermitian structure given by $(M, e)^{*}=$ $\left(M^{*}, e^{*}\right)$ and $\omega_{(M, e)}=e^{* *} \omega_{M} e \in \operatorname{Hom}_{\mathscr{C}^{\circ}}\left((M, e),\left(M^{* *}, e^{* *}\right)\right)$. Also, the functor $M \mapsto M^{\circ}$ is 1-hermitian and duality-preserving (the isomorphism $\left(M^{*}\right)^{\circ} \rightarrow\left(M^{\circ}\right)^{*}$ being $\left.\operatorname{id}_{M}\right)$, so we have a faithful and full functor $(M, s) \mapsto(M, s)^{\circ}=\left(M^{\circ}, s\right)$ from Sesq $(\mathscr{C})$ to Sesq $\left(\mathscr{C}^{\circ}\right)$. Henceforth, consider $\mathscr{C}$ and Sesq $(\mathscr{C})$ as full subcategories of $\mathscr{C}^{\circ}$ and Sesq $\left(\mathscr{C}^{\circ}\right)$, respectively; i.e., identify $M^{\circ}$ with $M$ and $(M, s)^{\circ}$ with $(M, s)$.

Lemma 5.17. Let $\mathscr{C}, \mathscr{C}^{\prime}$ be two hermitian categories and let $F: \mathscr{C} \rightarrow \mathscr{C}^{\prime}$ be an $\epsilon$-hermitian duality-preserving functor. Then:

(i) $F$ extends to an $\epsilon$-hermitian duality-preserving functor $F^{\circ}: \mathscr{C}^{\circ} \rightarrow \mathscr{C}^{\prime \circ}$. If $F$ is faithful and full, then so is $F^{\circ}$.

(ii) There is a 1-hermitian duality-preserving functor $G: \mathrm{A} \tilde{\mathbf{r}}_{2}(\mathscr{C})^{\circ} \rightarrow \mathrm{A} \tilde{\mathbf{r}}_{2}\left(\mathscr{C}^{\circ}\right)$. The functor $G$ fixes $\mathrm{Ar}_{2}(\mathscr{C})$ and induces an equivalence of categories.

Proof. (i) Define $F^{\circ}(M, e)=(F M, F e) \in \mathscr{C}^{\prime \circ}$. The rest is routine.

(ii) Let $G$ send $\left(\left(M, M^{\prime}, f, g\right),\left(e, e^{\prime \mathrm{op}}\right)\right) \in \mathrm{A}_{2}(\mathscr{C})^{\circ}$ to $\left((M, e),\left(M, e^{\prime}\right), e^{\prime *} f e, e^{\prime *} g e\right)$ and any morphism to itself. The details are left to the reader. 
Observe that the category $\mathscr{C}_{L}$ may not be pseudoabelian even when $\mathscr{C}$ is. We thus set $\mathscr{C}_{L}^{\circ}:=\left(\mathscr{C}_{L}\right)^{\circ}$.

Theorem 5.18. Let $(\mathscr{b}, *, \omega)$ be a pseudoabelian $K$-linear hermitian category such that $\operatorname{dim}_{K} \operatorname{Hom}\left(M, M^{\prime}\right)$ is finite for all $M, M^{\prime} \in \mathscr{C}$. Then the maps

$$
\mathrm{W}^{\epsilon}\left(\mathscr{R}_{L / K}\right): \mathrm{W}^{\epsilon}(\mathscr{C}) \rightarrow \mathrm{W}^{\epsilon}\left(\mathscr{C}_{L}^{\circ}\right) \text { and } \mathrm{W}\left(\mathscr{R}_{L / K}\right): \mathrm{W}_{S}(\mathscr{C}) \rightarrow \mathrm{W}_{S}\left(\mathscr{C}_{L}^{\circ}\right)
$$

are injective.

Proof. We begin by showing that $\mathrm{W}^{\epsilon}\left(\mathscr{R}_{L / K}\right): \mathrm{W}^{\epsilon}(\mathscr{C}) \rightarrow \mathrm{W}^{\epsilon}\left(\mathscr{C}_{L}^{\circ}\right)$ is injective. Let $(M, s) \in \mathrm{UH}^{\epsilon}(\mathscr{C})$ be such that $\left(M_{L}, s_{L}\right) \equiv 0$ in $\mathrm{W}^{\epsilon}\left(\mathscr{C}_{L}^{\circ}\right)$. Then there are objects $N, N^{\prime} \in \mathscr{C}_{L}^{\circ}$ such that $s_{L} \oplus \mathbb{H}_{N}^{\epsilon} \simeq \mathbb{H}_{N^{\prime}}^{\epsilon}$. Let

$$
(U, h)=(M, s) \oplus\left(N^{\prime} \oplus N^{\prime *}, \mathbb{H}_{N^{\prime}}^{\epsilon}\right) \quad \text { and } \quad E=\operatorname{End}_{\mathscr{C}^{\circ}}(U),
$$

and let $\sigma$ be the involution induced by $h$ on $E$. Set $E_{L}=E \otimes_{K} L=\operatorname{End}_{\mathscr{C}_{L}^{\circ}}\left(U_{L}\right)$ and $\sigma_{L}=\sigma \otimes_{K} \operatorname{id}_{L}$. Section 2E implies that $\mathscr{R}_{L / K}\left(\mathrm{~T}_{(U, h)}(M, s)\right)=\mathrm{T}_{\left(U_{L}, h_{L}\right)}\left(M_{L}, s_{L}\right) \equiv 0$ in $\mathrm{W}^{\epsilon}\left(E_{L}, \sigma_{L}\right)$, and by [Bayer-Fluckiger and Lenstra 1990, Proposition 1.2], this means $\mathrm{T}_{(U, h)}(M, s) \equiv 0$ in $\mathrm{W}^{\epsilon}(E, \sigma)$ (here we need $\left.\operatorname{dim}_{K} E<\infty\right)$. Since $\mathscr{C}$ is pseudoabelian, the map $\mathrm{T}_{(U, h)}: \mathscr{C}_{U} \rightarrow \mathscr{P}(E)$ is an equivalence of categories, hence the induced map $\mathrm{W}^{\epsilon}\left(\mathrm{T}_{(U, h)}\right): \mathrm{W}^{\epsilon}\left(\left.\mathscr{C}\right|_{U}\right) \rightarrow \mathrm{W}(\mathscr{P}(E))=\mathrm{W}^{\epsilon}(E, \sigma)$ is an isomorphism of groups. Therefore, $(M, s) \equiv 0$ in $\mathrm{W}^{\epsilon}\left(\left.\mathscr{C}\right|_{U}\right)$. In particular, the same identity holds in $\mathrm{W}^{\epsilon}(\mathscr{C})$.

Now let $(M, s) \in \operatorname{Sesq}(\mathscr{C})$ be such that $\left(M_{L}, s_{L}\right) \equiv 0$ in $\mathrm{W}_{S}\left(\mathscr{C}_{L}^{\circ}\right)$. Then by Proposition 5.13, $\left(M_{L}, s_{L}\right)$ is hyperbolic in $\mathscr{C}_{L}^{\circ}$ (but not, a priori, in $\mathscr{C}_{L}$ ). Let $F$ be the functor defined in Theorem 3.2 and let $J$ be the functor A $\tilde{r}_{2}\left(C_{C}\right)_{L} \rightarrow A \tilde{r}_{2}\left(\mathscr{C}_{L}\right)$ of Proposition 3.7. By the lemma, there is a fully faithful 1-hermitian dualitypreserving functor $J^{\prime}:=G J^{\circ}: A \tilde{\mathbf{r}}_{2}(\mathscr{C})_{L}^{\circ} \rightarrow A \tilde{r}_{2}\left(\mathscr{C}_{L}^{\circ}\right)$. Since $\left(M_{L}, s_{L}\right)$ is hyperbolic in $\mathscr{C}_{L}^{\circ}$, there is $Q \in \mathrm{A} \tilde{r}_{2}\left(\mathscr{C}_{L}^{\circ}\right)$ such that $F\left(M_{L}, s_{L}\right) \simeq\left(Q \oplus Q^{*}, \mathbb{M}_{Q}\right)$. Let $Z(M, s):=\left(M, M, s^{*} \omega_{M}, s\right)$ and $Z\left(M_{L}, s_{L}\right)=\left(M_{L}, M_{L}, s_{L}^{*} \omega_{M_{L}}, s_{L}\right)$. Recall that $F\left(M_{L}, s_{L}\right)=F \mathscr{R}_{L / K}(M, s)=J \mathscr{R}_{L / K} F(M, s)$ (Proposition 3.7) and hence $Q \oplus Q^{*} \simeq Z\left(M_{L}, s_{L}\right)=J\left(Z(M, s)_{L}\right)=J^{\prime}\left(Z(M, s)_{L}\right)$. As $J^{\prime}$ is fully faithful and its image is pseudoabelian, we may assume $Q=J^{\prime} H$ for some $H \in A \tilde{r}_{2}(\mathscr{C})_{L}^{\circ}$. We now have $J^{\prime}\left(H \oplus H^{*}, \mathbb{T}_{H}\right)=\left(Q \oplus Q^{*}, \mathbb{W}_{Q}\right) \simeq F\left(M_{L}, s_{L}\right)=J^{\prime} \mathscr{R}_{L / K} F(M, s)$, hence $\left(H \oplus H^{*}, \mathbb{H}_{H}\right) \simeq \mathscr{R}_{L / K} F(M, s)$ in $\mathrm{Ar}_{2}(\mathscr{C})_{L}^{\circ}$. In particular, $\mathscr{R}_{L / K} F(M, s) \equiv 0$ in $\mathrm{W}\left(\mathrm{A} \tilde{r}_{2}(\mathscr{C})_{L}^{\circ}\right)$. By the previous paragraph, this means $F(M, s) \equiv 0$ in $\mathrm{W}\left(\mathrm{A} \tilde{\mathbf{r}}_{2}(\mathscr{C})\right)$ and hence; $(M, s) \equiv 0$ in $\mathrm{W}_{S}(\mathscr{C})$.

We also have the following weaker version of Springer's theorem that works without assuming $\mathscr{C}$ is pseudoabelian:

Theorem 5.19. Suppose that $(\mathscr{C}, *, \omega)$ is a $K$-linear hermitian category such that $\operatorname{dim}_{K} \operatorname{Hom}\left(M, M^{\prime}\right)$ is finite for all $M, M^{\prime} \in \mathscr{C}$. Then $\mathrm{W}^{\epsilon}\left(\mathscr{R}_{L / K}\right): \mathrm{W}^{\epsilon}(\mathscr{C}) \rightarrow \mathrm{W}^{\epsilon}\left(\mathscr{C}_{L}\right)$ is injective. 
Proof. Let $(M, s) \in \mathrm{UH}^{\epsilon}(\mathscr{C})$ be such that $\left(M_{L}, s_{L}\right) \equiv 0$ in $\mathrm{W}^{\epsilon}\left(\mathscr{C}_{L}\right)$. Then there are objects $N_{L}, N_{L}^{\prime}$ such that $s_{L} \oplus \mathbb{U}_{N_{L}}^{\epsilon} \simeq \mathbb{H}_{N_{L}^{\prime}}^{\epsilon}$. Since $\mathbb{U}_{N_{L}}^{\epsilon}=\left(\mathbb{U}_{N}^{\epsilon}\right)_{L}$ and $\mathbb{U}_{N_{L}^{\prime}}^{\epsilon}=\left(\mathbb{H}_{N^{\prime}}^{\epsilon}\right)_{L}$, we have $\left(s \oplus \mathbb{U}_{N}^{\epsilon}\right)_{L} \simeq\left(\mathbb{H}_{N^{\prime}}^{\epsilon}\right)_{L}$. By Theorem 5.15, this means that $s \oplus \mathbb{U}_{N}^{\epsilon} \simeq \mathbb{H}_{N^{\prime}}^{\epsilon}$, hence $(M, s) \equiv 0$ in $\mathrm{W}^{\epsilon}(\mathscr{C})$.

5F. Weak Hasse principle. In this final subsection, we prove a version of the weak Hasse principle for systems of sesquilinear forms over hermitian categories. Recall that the weak Hasse principle asserts that two quadratic forms over a global field $k$ are isometric if and only if they are isometric over all completions of $k$. This actually fails for systems of quadratic forms, and we refer the reader to [Bayer-Fluckiger $1985 ; 1987]$ for necessary and sufficient conditions for the weak Hasse principle to hold in this case. A weak Hasse principle for sesquilinear forms defined over a skew field with a unitary involution was obtained in [Bayer-Fluckiger and Moldovan 2014].

Let $K$ be a commutative ring admitting an involution $\sigma$, and let $k$ be the fixed ring of $\sigma$. Let $\mathscr{C}$ be an additive $K$-category. A hermitian structure $(*, \omega)$ on $\mathscr{C}$ is called $(K, \sigma)$-linear if $(f a)^{*}=f^{*} \sigma(a)$ for all $a \in K$ and any morphism $f$ in $\mathscr{b}$. (This means that the functor $*$ is $k$-linear.) In this case, $\operatorname{End}(M)$ is a $K$-algebra for all $M \in \mathscr{C}$, and for any unimodular $\epsilon$-hermitian form $(M, s)$ over $\mathscr{C}$, the restriction of the involution $f \mapsto s^{-1} f^{*} s$ to $K \cdot \operatorname{id}_{M}$ is $\sigma$.

Suppose now that $K$ is a global field of characteristic not 2 admitting an involution $\sigma$ of the second kind with fixed field $k$, and that $\mathscr{C}$ admits a nonempty family of $(K, \sigma)$-linear hermitian structures $\left\{*_{i}, \omega_{i}\right\}_{i \in I}$. For every prime spot $p$ of $k$, let $k_{p}$ be the completion of $k$ at $p$, and set $K_{p}=K \otimes_{k} k_{p}, \sigma_{p}=\sigma \otimes_{k} \operatorname{id}_{k_{p}}$ and $\mathscr{b}_{p}=\mathscr{b}_{k} \otimes_{p}$. Then each of the hermitian structures $\left(*_{i}, \omega_{i}\right)$ gives rise to a $\left(K_{p}, \sigma_{p}\right)$-linear hermitian structure on $\mathscr{C}_{p}$, which we also denote by $\left(*_{i}, \omega_{i}\right)$.

Theorem 5.20. Let $K$ be a global field of characteristic not 2 admitting an involution $\sigma$ of the second kind with fixed field $k$. Let $\mathscr{b}$ be a $K$-category such that $\operatorname{dim}_{K} \operatorname{Hom}(M, N)$ is finite for all $M, N \in \mathscr{C}$, and assume there is a nonempty family $\left\{*_{i}, \omega_{i}\right\}_{i \in I}$ of $(K, \sigma)$-linear hermitian structures on $\mathscr{C}$. Then the weak Hasse principle (with respect to $k$ ) holds for systems of sesquilinear forms over $\left(\mathscr{C},\left\{*_{i}, \omega_{i}\right\}\right)$. That is, two systems of sesquilinear forms over $\left(\mathscr{C},\left\{*_{i}, \omega_{i}\right\}\right)$ are isometric if and only if they are isometric after applying $\mathscr{R}_{k_{p} / k}$ for all $p$.

We will need the following lemma. (The lemma seems to be known, but we could not find an explicit reference, and hence included here an ad hoc proof.)

Lemma 5.21. Let $L / K$ be any field extension, and let $\mathscr{C}$ be an additive $K$-category such that $\operatorname{dim}_{K} \operatorname{Hom}_{\mathscr{C}}(M, N)$ is finite for all $M, N \in \mathscr{C}$. Then for all $N, M \in \mathscr{C}$, we have $N \cong M$ if and only if $N_{L} \cong M_{L}$. 
Sketch of proof. By applying $\operatorname{Hom}_{\mathscr{C}}\left(M \oplus N,{ }_{-}\right)$, we may assume $M$ and $N$ are finitely generated projective right modules over $R:=\operatorname{End}(M \oplus N)$, which is a finite-dimensional $K$-algebra by assumption. Let $J$ be the Jacobson radical of $R$. By tensoring with $R / J$, we may assume $R$ is semisimple. Let $\left\{V_{i}\right\}_{i}$ be a complete list of the simple right $R$-modules and write

$$
\left(V_{i}\right)_{L}=\bigoplus_{j} W_{i j}^{n_{i j}}
$$

the $\left\{W_{i j}\right\}_{j}$ being pairwise nonisomorphic indecomposable $R_{L}$-modules. The $R_{L^{-}}$ modules $\left\{W_{i j}\right\}_{i, j}$ are pairwise nonisomorphic because $W_{i j}$ and $W_{i^{\prime} j^{\prime}}$ are nonisomorphic as $R$-modules when $i \neq i^{\prime}\left(W_{i j}\right.$ is isomorphic as an $R$-module to a direct sum of copies of $\left.V_{i}\right)$. Assume $M_{L} \cong N_{L}$ and write $M \cong \bigoplus_{i} V_{i}^{m_{i}}, N \cong \bigoplus_{i} V_{i}^{m_{i}^{\prime}}$. Then $\bigoplus_{i, j} W_{i j}^{m_{i} n_{i j}} \cong M_{L} \cong N_{L} \cong \bigoplus_{i, j} W_{i j}^{m_{i}^{\prime} n_{i j}}$. By the Krull-Schmidt theorem, we have $m_{i} n_{i j}=m_{i}^{\prime} n_{i j}$ for all $i, j$, hence $m_{i}=m_{i}^{\prime}$ and $M \cong N$.

Proof of Theorem 5.20. By Corollary 4.4, it is enough to verify the Hasse principle (with respect to $k$ ) for 1-hermitian forms in the category $\mathscr{G}:=\operatorname{Ar}_{2 I}(\mathscr{C})$. Our assumptions imply that $\mathscr{G}$ is a $(K, \sigma)$-linear category such that $\operatorname{dim}_{K} \operatorname{Hom}\left(Z, Z^{\prime}\right)$ is finite for all $Z, Z^{\prime} \in \mathscr{G}$. We now use the ideas developed in [Bayer-Fluckiger and Moldovan 2014, §9].

Let $(Z, h),\left(Z^{\prime}, h^{\prime}\right)$ be two unimodular 1-hermitian forms over $\mathscr{G}$ such that $\mathscr{R}_{k_{p} / k}(Z, h) \simeq \mathscr{R}_{k_{p} / k}\left(Z^{\prime}, h^{\prime}\right)$ for all $p$. By Lemma 5.21, this implies that $Z \cong Z^{\prime}$, so we may assume $Z=Z^{\prime}$.

Fix a 1-hermitian form $h_{0}$ on $Z$ and let $\tau$ be the involution induced by $h_{0}$ on $E:=\operatorname{End}(Z)$ (i.e., $\tau(x)=h_{0}^{-1} x^{*} h_{0}$ ). There is an equivalence relation on the elements of $E$ defined by $x \sim y$ if and only if there exists an invertible $z \in E$ such that $x=z y \tau(z)$. Let $H\left(\tau, E^{\times}\right)$be the set of equivalence classes of invertible elements $x \in E^{\times}$for which $x=\tau(x)$. In the same manner as in [Bayer-Fluckiger and Moldovan 2014, Theorem 5.1], we see that there is a one-to-one correspondence between isometry classes of unimodular 1-hermitian forms on $Z$ and elements $H\left(\tau, E^{\times}\right)$. It is given by $(Z, t) \mapsto h_{0}^{-1} t$.

Applying the same argument to $Z_{p}=\mathscr{R}_{k_{p} / k} Z \in \mathscr{G}_{p}$, we see that the weak Hasse principle is equivalent to the injectivity of the standard map

$$
\Phi: H\left(\tau, E^{\times}\right) \rightarrow \prod_{p} H\left(\tau_{p}, E_{p}^{\times}\right)
$$

where $E_{p}=\operatorname{End}\left(Z_{p}\right)=E \otimes_{k} k_{p}$ and $\tau_{p}=\tau \otimes_{k} \mathrm{id}_{k_{p}}$. Observe that since $\mathscr{G}_{\mathrm{G}}$ is $(K, \sigma)$-linear, $\tau$ is a unitary involution (and in fact, $\left.\left.\tau\right|_{K}=\sigma\right)$. By [Bayer-Fluckiger and Moldovan 2014, §9], this means that $\Phi$ is injective, hence the weak Hasse holds. 
Corollary 5.22. Let $K$ be a global field of characteristic not 2 admitting an involution $\sigma$ of the second kind with fixed field $k$. Let $A$ be a finite-dimensional $K$-algebra admitting a nonempty family of involutions $\left\{\sigma_{i}\right\}_{i \in I}$ such that $\left.\sigma_{i}\right|_{K}=\sigma$. Then the weak Hasse principle (with respect to $k$ ) holds for systems of sesquilinear forms $\operatorname{over}\left(A,\left\{\sigma_{i}\right\}\right)$.

\section{Acknowledgement}

Moldovan would like to thank Emmanuel Lequeu for many interesting and useful discussions.

\section{References}

[Bayer-Fluckiger 1985] E. Bayer-Fluckiger, "Intersections de groupes orthogonaux et principe de Hasse faible pour les systèmes de formes quadratiques sur un corps global", C. R. Acad. Sci. Paris Sér. I Math. 301:20 (1985), 911-914. MR 87a:11035 Zbl 0583.10013

[Bayer-Fluckiger 1987] E. Bayer-Fluckiger, "Principe de Hasse faible pour les systèmes de formes quadratiques", J. Reine Angew. Math. 378 (1987), 53-59. MR 88g:11015 Zbl 0625.10014

[Bayer-Fluckiger and Lenstra 1990] E. Bayer-Fluckiger and H. W. Lenstra, Jr., "Forms in odd degree extensions and self-dual normal bases", Amer. J. Math. 112:3 (1990), 359-373. MR 91h:11030 Zbl 0729.12006

[Bayer-Fluckiger and Moldovan 2014] E. Bayer-Fluckiger and D. A. Moldovan, "Sesquilinear forms over rings with involution", J. Pure Appl. Algebra 218:3 (2014), 417-423. MR 3124208 Zbl 06244924

[Bayer-Fluckiger et al. 1989] E. Bayer-Fluckiger, C. Kearton, and S. M. J. Wilson, "Hermitian forms in additive categories: finiteness results", J. Algebra 123:2 (1989), 336-350. MR 90k:11038 Zbl 0689.10031

[Björk 1971] J.-E. Björk, "Conditions which imply that subrings of semiprimary rings are semiprimary”, J. Algebra 19 (1971), 384-395. MR 44 \#1686 Zbl 0221.16019

[Camps and Dicks 1993] R. Camps and W. Dicks, "On semilocal rings", Israel J. Math. 81:1-2 (1993), 203-211. MR 94m:16027 Zbl 0802.16010

[Fainsilber 1994] L. Fainsilber, "Formes Hermitiennes sur des algèbres sur des anneaux locaux", Publ. Math. Besançon Algèbr. Théor. Nombres 1994 (1994). MR 96c:11041 Zbl 0880.11036

[First 2013] U. A. First, "Semi-invariant subrings", J. Algebra 378 (2013), 103-132. MR 3017016 Zbl 1282.16043

[Hinohara 1960] Y. Hinohara, "Note on non-commutative semi-local rings", Nagoya Math. J. 17 (1960), 161-166. MR 24 \#A2595 Zbl 0109.26501

[Karoubi 1978] M. Karoubi, K-theory: an introduction, Grundlehren der Mathematischen Wissenschaften 226, Springer, Berlin, 1978. MR 58 \#7605 Zbl 0382.55002

[Keller 1988] B. Keller, "A remark on quadratic spaces over noncommutative semilocal rings", Math. Z. 198:1 (1988), 63-71. MR 89i:11055 Zbl 0653.10021

[Knebusch 1969] M. Knebusch, “Isometrien über semilokalen Ringen”, Math. Z. 108 (1969), 255268. MR 40 \#5602 Zbl 0188.35502

[Knus 1991] M.-A. Knus, Quadratic and Hermitian forms over rings, Grundlehren der Mathematischen Wissenschaften 294, Springer, Berlin, 1991. MR 92i:11039 Zbl 0756.11008 
[Quebbemann et al. 1979] H.-G. Quebbemann, W. Scharlau, and M. Schulte, "Quadratic and Hermitian forms in additive and abelian categories", J. Algebra 59:2 (1979), 264-289. MR 82d:18015 Zbl 0412.18016

[Reiter 1975] H. Reiter, "Witt's theorem for noncommutative semilocal rings", J. Algebra 35 (1975), 483-499. MR 52 \#8119 Zbl 0306.16017

[Riehm 1974] C. Riehm, “The equivalence of bilinear forms”, J. Algebra 31 (1974), 45-66. MR 50 \#368 Zbl 0283.15016

[Riehm and Shrader-Frechette 1976] C. Riehm and M. A. Shrader-Frechette, "The equivalence of sesquilinear forms", J. Algebra 42:2 (1976), 495-530. MR 55 \#380 Zbl 0347.15008

[Rowen 1988] L. H. Rowen, Ring theory, vol. 1, Pure and Applied Mathematics 127, Academic Press, Boston, 1988. MR 89h:16001 Zbl 0651.16001

[Scharlau 1985] W. Scharlau, Quadratic and Hermitian forms, Grundlehren der Mathematischen Wissenschaften 270, Springer, Berlin, 1985. MR 86k:11022 Zbl 0584.10010

Received May 6, 2013. Revised December 3, 2013.

EVA BAYER-FLUCKIGER

École Polytechnique FÉdÉRAle de Lausanne

SB MATHGEOM CSAG

BÂTIMENT MA

STATION 8

CH-1015 LAUSANNE

SWITZERLAND

eva.bayer@epfl.ch

URIYA A. FIRST

EINSTEIN INSTITUTE OF MATHEMATICS

HEBREW UNIVERSITY OF JERUSALEM

EDWARD J. SAFRA CAMPUS

Givat RAM, 91904, JERUSALEM

ISRAEL

uriya.first@gmail.com

DANiEl A. Moldovan

École Polytechnique Fédérale de LAUSANNe

AVEnue DE Morges 88

CH-1004 LAUSANNE

SWITZERLAND

mda@gmail.com 


\title{
PACIFIC JOURNAL OF MATHEMATICS
}

\author{
msp.org/pjm
}

Founded in 1951 by E. F. Beckenbach (1906-1982) and F. Wolf (1904-1989)

\section{EDITORS}

Don Blasius (Managing Editor)

Department of Mathematics

University of California

Los Angeles, CA 90095-1555

blasius@math.ucla.edu

\author{
Paul Balmer \\ Department of Mathematics \\ University of California \\ Los Angeles, CA 90095-1555 \\ balmer@math.ucla.edu \\ Robert Finn \\ Department of Mathematics \\ Stanford University \\ Stanford, CA 94305-2125 \\ finn@math.stanford.edu \\ Sorin Popa \\ Department of Mathematics \\ University of California \\ Los Angeles, CA 90095-1555 \\ popa@math.ucla.edu
}

\author{
Vyjayanthi Chari \\ Department of Mathematics \\ University of California \\ Riverside, CA 92521-0135 \\ chari@math.ucr.edu \\ Kefeng Liu \\ Department of Mathematics \\ University of California \\ Los Angeles, CA 90095-1555 \\ liu@math.ucla.edu \\ Jie Qing \\ Department of Mathematics \\ University of California \\ Santa Cruz, CA 95064 \\ qing@ cats.ucsc.edu
}

\section{PRODUCTION}

Silvio Levy, Scientific Editor, production@msp.org

\section{SUPPORTING INSTITUTIONS}

ACADEMIA SINICA, TAIPEI

CALIFORNIA INST. OF TECHNOLOGY

INST. DE MATEMÁTICA PURA E APLICADA

KEIO UNIVERSITY

MATH. SCIENCES RESEARCH INSTITUTE

NEW MEXICO STATE UNIV.

OREGON STATE UNIV.

\author{
STANFORD UNIVERSITY \\ UNIV. OF BRITISH COLUMBIA \\ UNIV. OF CALIFORNIA, BERKELEY \\ UNIV. OF CALIFORNIA, DAVIS \\ UNIV. OF CALIFORNIA, LOS ANGELES \\ UNIV. OF CALIFORNIA, RIVERSIDE \\ UNIV. OF CALIFORNIA, SAN DIEGO \\ UNIV. OF CALIF., SANTA BARBARA
}

\author{
Daryl Cooper \\ Department of Mathematics \\ University of California \\ Santa Barbara, CA 93106-3080 \\ cooper@math.ucsb.edu \\ Jiang-Hua Lu \\ Department of Mathematics \\ The University of Hong Kong \\ Pokfulam Rd., Hong Kong \\ jhlu@maths.hku.hk \\ Paul Yang \\ Department of Mathematics \\ Princeton University \\ Princeton NJ 08544-1000 \\ yang@math.princeton.edu
}

These supporting institutions contribute to the cost of publication of this Journal, but they are not owners or publishers and have no responsibility for its contents or policies.

See inside back cover or msp.org/pjm for submission instructions.

The subscription price for 2014 is US $\$ 410 /$ year for the electronic version, and \$535/year for print and electronic.

Subscriptions, requests for back issues and changes of subscribers address should be sent to Pacific Journal of Mathematics, P.O. Box 4163, Berkeley, CA 94704-0163, U.S.A. The Pacific Journal of Mathematics is indexed by Mathematical Reviews, Zentralblatt MATH, PASCAL CNRS Index, Referativnyi Zhurnal, Current Mathematical Publications and Web of Knowledge (Science Citation Index).

The Pacific Journal of Mathematics (ISSN 0030-8730) at the University of California, c/o Department of Mathematics, 798 Evans Hall \#3840, Berkeley, CA 94720-3840, is published twelve times a year. Periodical rate postage paid at Berkeley, CA 94704, and additional mailing offices. POSTMASTER: send address changes to Pacific Journal of Mathematics, P.O. Box 4163, Berkeley, CA 94704-0163.

PJM peer review and production are managed by EditFLOW ${ }^{\circledR}$ from Mathematical Sciences Publishers.

\section{PUBLISHED BY}

\section{mathematical sciences publishers \\ nonprofit scientific publishing}

http://msp.org/

(C) 2014 Mathematical Sciences Publishers 


\section{PACIFIC JOURNAL OF MATHEMATICS}

Volume $270 \quad$ No. $1 \quad$ July 2014

Hermitian categories, extension of scalars and systems of sesquilinear forms

EVA BAYER-FLUCKIGER, URIYA A. FIRST and DANIEL A.

MOLDOVAN

Realizations of the three-point Lie algebra $\mathfrak{s l}(2, \mathscr{R}) \oplus\left(\Omega_{\mathscr{R}} / d \mathscr{R}\right)$

BEN COX and ELIZABETH JURISICH

Multi-bump bound state solutions for the quasilinear Schrödinger equation with critical frequency

YUXIA GUO and ZHONGWEI TANG

On stable solutions of the biharmonic problem with polynomial growth

Hatem Hajlaoui, Abdellaziz Harrabi and Dong Ye

Valuative multiplier ideals

ZHENGYU HU

Quasiconformal conjugacy classes of parabolic isometries of complex hyperbolic space

YOUNGJU KIM

On the distributional Hessian of the distance function

Carlo MantegazZa, Giovanni Mascellani and Gennady URALTSEV

Noether's problem for abelian extensions of cyclic $p$-groups

IVO M. MICHAILOV

Legendrian $\theta$-graphs

Danielle O’Donnol and Elena PaVelescu

A class of Neumann problems arising in conformal geometry

WEIMIN SHENG and LI-XIA YUAN

Ryshkov domains of reductive algebraic groups

TAKAO WATANABE 